\title{
A Single-Sorted Theory of Multisets
}

\author{
Hoang-Vu Dang
}

\begin{abstract}
An axiomatic account of multiset theory is given, where multiplicities are of the same sort as sets (with their own internal structures). Various theories are proposed covering different existing multiset systems, as well as a stronger theory which is equiconsistent with Zermelo-Fraenkel set theory and with antifoundation. The inclusion relation receives a recursive definition in terms of membership and is shown to be not always antisymmetric.
\end{abstract}

\section{Introduction}

Multisets are sets with possibly repeated elements, somewhat natural objects that arise in various situations in both mathematics and computing. However despite numerous accounts of multisets, some by quite well-known mathematicians, there has been no consensus on how to axiomatize them. The best survey of these accounts is Wayne Blizard's "The development of multiset theory" [4], and the two most comprehensive proposals seem to be Blizard's own in [2] and [5], the latter even allowing for infinite multiplicities. However, like other multiset theories, they are both twosorted theories where the multiplicities are different types of objects from the multisets they support. This means having separate axioms for multiplicity arithmetic, and in the infinite case it assumes a predefined model of cardinal arithmetic; for example, [5] uses cardinals in a model of Zermelo-Fraenkel (ZF) set theory.

Here we will propose a one-sorted account of multisets, where multiplicities and sets come from the same domain and follow the same axioms. In this system, multiplicities are no longer predefined cardinal numbers but are also multisets with their own internal structures. The natural ordering of multiplicities will be identified with the subset relation; that is, intuitively we consider $x$ to be less than $y$ as multiplicities if $x$ is a proper subset of $y$. The axioms we propose will mirror Zermelo-Fraenkel set theory, mutatis mutandis, the only real complication coming from the subset relation for multisets (which becomes naturally recursive upon being identified with

Received March 7, 2012; accepted October 6, 2012

2010 Mathematics Subject Classification: Primary 03E75

Keywords: multiset, axiomatic, antifoundation

(C) 2014 by University of Notre Dame 10.1215/00294527-2688042 
the ordering on multiplicities). Using a graph-based model closely related to the construction in D'Agostino and Visser [6], we prove the consistency of our multiset theory with antifoundation and show that unlike in set theory, the subset relation on multisets under our definition need not be antisymmetric; hence an extra axiom is necessary if one wants to enforce the antisymmetry of the inclusion relation.

\section{Some Notes on Notation}

For clarity we keep the number of brackets to a minimum and assign descending priorities to the following symbols:

- universal and existential quantifiers and their variants,

- conjunction and disjunction,

- implications.

If $R$ is a binary relation or binary predicate symbol, write

$$
\begin{gathered}
(\forall x R y) \varphi(x) \Leftrightarrow_{d f}(\forall x)(x R y \Rightarrow \varphi(x)), \\
(\exists x R y) \varphi(x) \Leftrightarrow_{d f}(\exists x)(x R y \wedge \varphi(x)) .
\end{gathered}
$$

Similarly, write $\left(\forall\left\langle x_{1} \cdots x_{n}\right\rangle R y\right) \varphi\left(x_{1} \cdots x_{n}\right)$ and $\left(\exists\left\langle x_{1} \cdots x_{n}\right\rangle R y\right) \varphi\left(x_{1} \cdots x_{n}\right)$ with the obvious meanings.

Relations and functions mean sets of ordered pairs (or tuples in the case of higher arity) unless specified otherwise. Write $f \in$ Function to mean that $f$ is a function and $f^{-1}$ for the inverse $\{\langle y, x\rangle:\langle x, y\rangle \in f\}$ (which may not be a function). Write dom $f$ for the domain of the function $f$ and $\operatorname{ran} f$ for the range of $f$.

Write $R \in$ Relation to mean that $R$ is a relation, and write Dom $R$ for the relational domain (or field) of $R$, that is, the set $\{x:(\exists y)(\langle x, y\rangle \in R \vee\langle y, x\rangle \in R)\}$.

Remark 2.1 For any function $f$, we have Dom $f=\operatorname{dom} f \cup \operatorname{ran} f$.

\section{The Theory}

This is a one-sorted theory where the same variables will be used for multisets and multiplicities; we may call them sets where there is no danger of confusion with traditional sets. The membership predicate $x \epsilon_{a} y$ means that $x$ belongs to $y$ with multiplicity $a$.

Definition 3.1 The language of multisets $\mathscr{L}_{\mathcal{M}}$ has one sort of variable and two predicate symbols: the identity $=$ and the ternary symbol $\in$. (In practice we write $x \in_{a} y$.)

Remark 3.2 The membership and subset relations we will define on multisets will be denoted by overlined symbols to differentiate them from their set-theoretic counterparts. This distinction is especially important when we build a model for our multiset theory from a model of set theory.

Definition 3.3 We write $x \bar{\epsilon} y$ for $(\exists a) x \epsilon_{a} y$, that is, $x$ belongs to $y$.

Axiom 3.0.1 (Axiom of unique multiplicity) We have

$$
(\forall x, y, a, b)\left(x \in_{a} y \wedge x \in_{b} y \Rightarrow a=b\right) .
$$

Definition 3.4 We write $\frac{y}{x}$ for the unique multiplicity of $x$ in $y$. 


\section{Axiom 3.0.2 (Axiom of extensionality) We have}

$$
(\forall x, y)\left(x=y \Leftrightarrow(\forall a, b)\left(a \epsilon_{b} x \Leftrightarrow a \epsilon_{b} y\right)\right) .
$$

Definition 3.5 If $\varphi(x, y)$ has two free variables and possibly parameters such that $(\forall x)(\exists ! y) \varphi(x, y)$, we say that $\varphi$ defines a function class on multisets.

Axiom 3.0.3 (Axiom of empty set) We have

$$
(\exists x)(\forall y) y \notin x .
$$

Extensionality ensures that the empty multiset, which we denote by $\bar{\emptyset}$, is unique.

\section{Axiom Schema 3.0.4 (Axiom schema of comprehension) We have}

$$
(\forall x)(\exists y)(\forall z, b)\left(z \epsilon_{b} y \Leftrightarrow z \epsilon_{b} x \wedge \varphi(z, b)\right)
$$

for all formulas $\varphi$ with two free variables and possibly parameters.

Note that the set given by comprehension inherits the multiplicities from the original set. By introducing axioms that deal with multiplicities separately, we can extend the basic theory to incorporate different systems of multisets, for example, those with only finite multiplicities or cardinal multiplicities.

Definition 3.6 Let $\varphi$ define a function class on multisets.

Write $\{x \otimes y: \varphi(x, y)\}$ for the multiset $a$ satisfying $(\forall x, y)\left(x \in_{y} a \Leftrightarrow \varphi(x, y)\right)$ (i.e., $a$ contains $x$ with multiplicity $y$ if and only if $\varphi(x, y)$ holds) if such a multiset exists.

As a special case, for any concrete natural number $\mathbf{n}$ we write $\left\{x_{1} \otimes y_{1}, \ldots\right.$, $\left.x_{\mathbf{n}} \otimes y_{\mathbf{n}}\right\}$ for the multiset $a$ satisfying the following if it exists (though later with all of our axioms, such multisets do indeed exist):

$$
(\forall x, y)\left(x \in_{y} a \Leftrightarrow\left(x=x_{1} \wedge y=y_{1}\right) \vee \cdots \vee\left(x=x_{\mathbf{n}} \wedge y=y_{\mathbf{n}}\right)\right) .
$$

The multisets specified in the definition above are unique by extensionality.

Axiom 3.0.5 (Axiom of pairing) We have

$$
(\forall x, y)(\exists a) a=\{x \otimes \bar{\emptyset}, y \otimes \bar{\emptyset}\} .
$$

Definition 3.7 We have an ordered pair

$$
\langle x, y\rangle:=\{\{x \otimes \bar{\emptyset}\} \otimes \bar{\emptyset},\{x \otimes \bar{\emptyset}, y \otimes \bar{\emptyset}\} \otimes \bar{\emptyset}\} .
$$

3.1 The subset relation Intuitively in our theory there should be an ordering on multiplicities (namely, there are more copies of something than of another). With this in mind, we regard a multiset $x$ as a subset of $y$ if and only if every member of $x$ appears in $y$ with greater or equal multiplicity. As mentioned before, identifying the subset relation with the ordering on multiplicities gives us a natural recursive definition of subset.

Axiom Schema 3.1.1 The relation $\overline{\mathrm{C}}$ is a partial order and the largest class relation such that

$$
(\forall x, y)\left(x \bar{\subset} y \Leftrightarrow(\forall a \bar{\epsilon} x)\left(a \bar{\epsilon} y \wedge \frac{x}{a} \bar{\subset} \frac{y}{a}\right)\right) .
$$

In other words, if $\varphi(x, y)$ is a formula (possibly with parameters) such that

$$
(\forall x, y)\left(\varphi(x, y) \Leftrightarrow(\forall a \bar{\epsilon} x)\left(a \bar{\epsilon} y \wedge \varphi\left(\frac{x}{a}, \frac{y}{a}\right)\right)\right),
$$

then $(\forall x, y)(\varphi(x, y) \Rightarrow x \bar{\subset} y)$. 
We will prove this schema from the other axioms by formally defining $x \bar{\subset} y$ as shorthand for the formula below. It trivially follows from that definition that if $x \bar{c} y$, then every member of $x$ is a member of $y$. Furthermore, $\bar{\emptyset}$ is a subset of everything, while nothing else is a subset of $\bar{\emptyset}$.

Definition 3.8 We have

$$
\begin{aligned}
x \bar{\subset} y \Leftrightarrow_{d f} x=y & \vee(\exists R)(\langle x, y\rangle \bar{\epsilon} R \\
& \left.\wedge(\forall v, w)\left(\langle v, w\rangle \bar{\epsilon} R \Rightarrow(\forall a \bar{\epsilon} v)\left(a \bar{\epsilon} w \wedge\left\langle\frac{v}{a}, \frac{w}{a}\right\rangle \bar{\epsilon} R\right)\right)\right) .
\end{aligned}
$$

Lemma 3.9 We have $(\forall x)(\forall y)(x \bar{\epsilon} y \Leftrightarrow\{x \otimes \bar{\emptyset}\} \bar{\subset} y)$.

Proof In one direction $\{x \otimes \bar{\emptyset}\} \bar{\subset} y$ since $\bar{\emptyset}$ is a subset of everything; the other direction is trivial.

Axiom 3.1.2 (Axiom of subset) We have

$$
(\forall x, y)(x \bar{\subset} y \wedge y \bar{\subset} x \Rightarrow x=y) .
$$

Remark 3.10 At this point one may question the necessity of this axiom, as the subset relation in set theory is trivially antisymmetric. However, in the last section we will show that the axiom of subset is independent from the remaining multiset axioms by means of a syntactic model.

We define the union of $x$ to be the minimal superset of all members of $x$. In the context of two-sorted multiset theory, this definition corresponds to taking the supremum of multiplicities of the same object, as opposed to what [2] calls the additive union where multiplicities are added.

Axiom 3.1.3 (Axiom of union) We have

$$
(\forall x)(\exists b)(\forall a)(b \bar{\subset} a \Leftrightarrow(\forall y \bar{\epsilon} x) y \bar{\subset} a) .
$$

Following set theory convention, we denote the union of $x$ by $\bigcup x$ and write $x \cup y$ for $\bigcup\{x \otimes \bar{\emptyset}, y \otimes \bar{\emptyset}\}$. Since $\bar{C}$ is antisymmetric, $\bigcup x$ is unique for every $x$.

We follow the same approach in defining replacement. Let the formula $\varphi$ define a function on $x$, and let $a$ be in the image of $x$. If $a$ is the image of more than one $y \bar{\epsilon} x$, let the multiplicity of $a$ be the $\overline{\mathcal{C}}$-least upper bound (i.e., the union) of $\frac{x}{y}$ for all preimages $y$ of $a$. If $y$ is unique, it easily follows that the multiplicity of $a$ is $\frac{x}{y}$ since $\bar{C}$ is both reflexive and antisymmetric.

Axiom Schema 3.1.4 (Axiom schema of replacement) We have

$$
\begin{aligned}
& (\forall x)\left(( \forall a \overline { \epsilon } x ) ( \exists ! b ) \varphi ( a , b ) \Rightarrow ( \exists y ) ( \forall b , d ) \left(b \in_{d} y\right.\right. \\
& \left.\left.\quad \Leftrightarrow(\exists a \bar{\epsilon} x) \varphi(a, b) \wedge(\forall e)\left(d \bar{\subset} e \Leftrightarrow(\forall a \bar{\epsilon} x)\left(\varphi(a, b) \Rightarrow \frac{x}{a} \bar{\subset} e\right)\right)\right)\right)
\end{aligned}
$$

for all formulas $\varphi$ with two free variables and possibly with parameters.

Definition 3.11 It is clear that the multiset given by replacement is unique for each $x$ and each function class $\varphi$, and we will denote it by $\operatorname{Rep}_{\varphi} x$.

Lemma 3.12 We have $(\forall x, b)(b=\bigcup x \Rightarrow(\forall a)(a \bar{\epsilon} b \Leftrightarrow(\exists y \bar{\epsilon} x) a \bar{\epsilon} y))$. 
Proof If $(\forall y \bar{\epsilon} x) a \notin y$, by comprehension let $z:=\left\{v \otimes w: v \epsilon_{w} b \wedge v \neq a\right\}$.

Let $y \bar{\epsilon} x$, and let $R$ be a witness to $y \bar{\subset} b$. Let $A:=\bigcup\{R \otimes \bar{\emptyset},\{\langle y, z\rangle \otimes \bar{\emptyset}\} \otimes \bar{\emptyset}\}$, and define by comprehension from $A$ the multiset

$$
S:=\left\{v \otimes w: v \epsilon_{w} A \wedge(v \bar{\epsilon} R \vee v=\langle y, z\rangle)\right\} .
$$

As a binary relation, $S$ is obtained by adding $\langle y, z\rangle$ to $R$. Since $a \bar{\notin} y$,

$$
(\forall\langle v, w\rangle \bar{\epsilon} S)(\forall c \bar{\epsilon} v)\left(c \bar{\epsilon} w \wedge\left\langle\frac{v}{c}, \frac{w}{c}\right\rangle \bar{\epsilon} S\right) .
$$

Hence $S$ is a witness to $y \bar{\subset} z$, and thus $b \bar{\subset} z$ by definition of union, so $a \notin b$.

Conversely, let $y \bar{\epsilon} x$ and $a \bar{\epsilon} y$; then $a \bar{\epsilon} b$ since $y \bar{\subset} b$ by definition.

We will prove later that the multiplicity of $a \bar{\epsilon} \cup x$ is the union of multiplicities of $a$ in all $b \bar{\epsilon} x$, using the schema of multiplicity replacement.

Definition 3.13 The canonical power set of $x$ is $\mathcal{P} x:=\{y \otimes \bar{\emptyset}: y \bar{C} x\}$.

Axiom 3.1.5 (Axiom of power set) We have

$$
(\forall x)(\exists y) y=\mathcal{P} x .
$$

Lemma 3.14 For any $x, y$ there exists the product of $x$ and $y$, namely,

$$
x \times y:=\{\langle v, w\rangle \otimes \bar{\emptyset}: v \bar{\epsilon} x \wedge w \bar{\epsilon} y\} .
$$

Proof This is proved by comprehension from $\mathcal{P}^{3}(x \cup y)$.

Lemma 3.15 The relation $\bar{\subset}$ is transitive and thus a partial order.

Proof Suppose $a \bar{c} b$ and $b \bar{c} c$. If $a=b$ or $b=c$ the proof is trivial, so suppose that they are distinct. Let $R_{1}$ witness $a \bar{C} b$, and let $R_{2}$ witness $b \bar{\subset} c$; then the following relation witnesses $a \bar{C} c$ :

$$
R:=\left\{\langle x, z\rangle \otimes \bar{\emptyset}:(\exists y)\left(\langle x, y\rangle \bar{\epsilon} R_{1} \wedge\langle y, z\rangle \bar{\epsilon} R_{2}\right)\right\} .
$$

Lemma 3.16 (Intersection) We have

$$
(\exists x) \varphi(x) \Rightarrow(\exists b)(\forall a)(a \bar{\subset} b \Leftrightarrow(\forall y)(\varphi(y) \Rightarrow a \bar{\subset} y))
$$

for any formula $\varphi$ with one free variable and possibly parameters.

Proof Let $\varphi(x)$ hold for some $x$. By comprehension from $\mathcal{P} x$ and union we have the multiset

$$
b:=\bigcup\{v \otimes \bar{\emptyset}: v \bar{\subset} x \wedge(\forall y)(\varphi(y) \Rightarrow v \bar{\subset} y)\} .
$$

If $\varphi(y)$ holds, then $v \bar{\subset} y$ for any $v$ in the multiset whose union is $b$; hence $b \bar{\subset} y$ by the definition of union. Therefore $(\forall a \bar{C} b) a \bar{\subset} y$ by transitivity of $\bar{C}$. Conversely, if $a \bar{C} y$ for all $y$ such that $\varphi(y)$ holds, then $a \bar{C} x$. Hence $a$ is in the union, so $a \bar{\subset} b$.

For convenience we denote the intersection as defined in Lemma 3.16 by $\bigcap_{\varphi(x)} x$. The intersection is unique if it exists since $\bar{C}$ is antisymmetric. If $\varphi(x)$ is the formula $x \bar{\epsilon} y$, we simply write $\bigcap y$. If $\varphi(x)$ is the formula $x \bar{\epsilon} a \wedge x \bar{\epsilon} b$, we write $a \cap b$. 
Remark 3.17 Normally one would expect $x \epsilon_{\bar{\emptyset}} y$ to denote nonmembership, since it fits with the intuition of $x$ belonging to $y$ zero times. However, that would give rise to odd phenomena, for example, nonempty multisets with the same members but empty intersection. Suppose that $x$ and $y$ are nonempty and disjoint, which always exist if the model has more than one nonempty object. Then $\{\bar{\emptyset} \otimes x\}$ and $\{\bar{\emptyset} \otimes y\}$ have the same member, namely $\bar{\emptyset}$, but the multiplicity of $\bar{\emptyset}$ in the intersection must be empty since it is a subset of both $x$ and $y$.

Remark 3.18 There is no negative membership in our theory since $\bar{\emptyset}$ is already the bottom multiplicity. (Recall that $\bar{\emptyset}$ is a subset of everything, and we already chose to identify the ordering of multiplicities with the subset relation on multisets.) For a quick overview of negative multiplicities and a theory of multisets with integer multiplicities (including the negative integers), see Blizard [3].

\subsection{Relations and functions}

Definition 3.19 A multiset $R$ is a (binary) relation if all its members are ordered pairs. Define the canonical domain (or field) of $R$ by comprehension as

$$
\operatorname{Dom} R:=\left\{x \otimes v:(\exists y)(\langle x, y\rangle \bar{\epsilon} R \vee\langle y, x\rangle \bar{\epsilon} R) \wedge x \epsilon_{v} \bigcup^{2} R\right\} .
$$

Definition 3.20 A relation $f$ is a function if

$$
(\forall a, x, y)((\langle a, x\rangle \bar{\epsilon} f \wedge\langle a, y\rangle \bar{\epsilon} f) \Rightarrow x=y) .
$$

We define the canonical domain and range of a function $f$ as

$$
\begin{aligned}
\operatorname{dom} f: & =\left\{x \otimes v:(\exists y)\langle x, y\rangle \bar{\epsilon} f \wedge x \epsilon_{v} \bigcup^{2} f\right\}, \\
\operatorname{ran} f: & =\left\{y \otimes v:(\exists x)\langle x, y\rangle \bar{\epsilon} f \wedge y \epsilon_{v} \bigcup^{2} f\right\} .
\end{aligned}
$$

Note that if $f$ is a function, then Dom $f=\operatorname{dom} f \cup \operatorname{ran} f$. In general, $f$ can be regarded as a function on any multiset with the same members as dom $f$. Intuitively when viewed from outside the model of our theory, a function is just a map which sends multisets to multisets; the multiplicities in the domain and in the graph of the function itself are thus of no importance.

For convenience we also extend our notation with $\{f(x) \otimes g(y): \varphi(x, y)\}$ where $f, g$ are either functions or formulas defining function classes on multisets, with the obvious meaning.

\subsection{Well-orders and infinity}

Definition 3.21 Say that $X$ is a closed multiset, and write $X \bar{\epsilon}$ Closed if

$$
(\forall v \bar{\epsilon} X)(\forall a, b)\left(a \epsilon_{b} v \Rightarrow(a \bar{\epsilon} X \wedge b \bar{\epsilon} X)\right) .
$$

Definition 3.22 A relation $R$ is a well-order if it is a total order and any nonempty multiset $A \bar{C}$ Dom $R$ has an $R$-minimal member.

For the axiom of infinity we define an analogue of the von Neumann $\omega$.

Definition 3.23 Write $\alpha \in O N$ for the formula saying both of the following:

- $(\forall x \bar{\epsilon} \alpha)\left(\frac{\alpha}{x}=\bar{\emptyset} \wedge(\forall y \bar{\epsilon} x)\left(y \bar{\epsilon} \alpha \wedge \frac{x}{y}=\bar{\emptyset}\right)\right)$,

- the relation $\bar{\epsilon}$ restricted to $\alpha$ is a well-order. 
Write $\alpha^{+}$for $\alpha \cup\{\alpha \otimes \bar{\emptyset}\}$, and write $\alpha<\beta$ for $\alpha \bar{\epsilon} \beta$ when $\alpha$ and $\beta$ are both ordinals.

Lemma 3.24 If $\alpha \in O N$, then $\alpha$ is closed. Furthermore, if $\beta \bar{\epsilon} \alpha$, then $\beta \in O N$.

Proof For $\alpha$ to be closed it suffices to show $\bar{\emptyset} \bar{\epsilon} a$, but the $\bar{\epsilon}$-minimal member of $\alpha$ has to be empty.

Suppose $\beta \bar{\epsilon} \alpha$ and $x \bar{\epsilon} \beta$; then $\frac{\beta}{x}=\bar{\emptyset}$ since $\alpha \in O N$. But $x \bar{\epsilon} \alpha$, so for any $y \bar{\epsilon} x$ we have $y \bar{\epsilon} \alpha$ and $\frac{x}{y}=\bar{\emptyset}$. Now $\bar{\epsilon}$ well-orders $\alpha$, and $\beta \bar{\epsilon} y$ would violate well-foundedness of $\bar{\epsilon}$ on $\alpha$, so $y \bar{\epsilon} \beta$. To prove that $\bar{\epsilon}$ well-orders $\beta$, note that every member of $\beta$ is a member of $\alpha$ and $\bar{\epsilon}$ well-orders $\alpha$.

Remark 3.25 It follows directly from the definition of $\alpha^{+}$that $\alpha \epsilon_{\bar{\emptyset}} \alpha^{+}$(note that $\alpha$ cannot be a member of itself due to the well-ordering condition in the definition of ordinals), and $\alpha^{+} \in O N$ whenever $\alpha \in O N$.

Axiom 3.3.1 (Axiom of infinity) We have

$$
(\exists x \in O N)\left(\bar{\emptyset} \epsilon_{\bar{\emptyset}} x \wedge(\forall y \bar{\epsilon} x)\left(\left(y=\bar{\emptyset} \vee(\exists z \bar{\epsilon} x) y=z^{+}\right) \wedge y^{+} \epsilon_{\bar{\emptyset}} x\right)\right) .
$$

Definition 3.26 We call this multiset $\overline{\boldsymbol{\omega}}$ and use the usual numerals to stand for the appropriate finite ordinals; that is, $n+1$ stands for $n^{+}$.

Remark 3.27 The usual schema of induction on the ordinals also works here since multiset ordinals are defined to be closed.

\subsection{The maximal property of $\bar{C}$}

Lemma 3.28 For any $x$, there exists a closed multiset with $x$ as a member.

Proof Define $\psi(v, w) \Leftrightarrow d f w=v \cup\left\{b \otimes b:(\exists a) a \epsilon_{b} v\right\}$.

By union and replacement $(\forall v)(\exists w) \psi(v, w)$.

Define a function class $\varphi(x, y)$ from $\bar{\omega}$ to the universe of multisets as follows:

$\varphi(x, y) \Leftrightarrow_{d f}(\exists f \in$ Function $)(f(x)=y \wedge f(0)=\{x \otimes \bar{\emptyset}\} \wedge \operatorname{dom} f \bar{\subset} \bar{\omega}$ $\left.\wedge(\forall n \bar{\epsilon} \operatorname{dom} f)(\forall m<n) m \bar{\epsilon} \operatorname{dom} f \wedge(\forall n \bar{\epsilon} \operatorname{dom} f) f(n+1)=\bigcup \operatorname{Rep}_{\psi} f(n)\right)$.

The formula $\varphi$ defines a function on all of $\overline{\boldsymbol{\omega}}$ : if there is $n \bar{\epsilon} \overline{\boldsymbol{\omega}}$ such that $(\nexists y) \varphi(n, y)$, without loss of generality assume that $n$ is $\bar{\epsilon}$-minimal. Then $n \neq 0$, so $n=m+1$ for some $m \overline{\boldsymbol{\epsilon}} \overline{\boldsymbol{\omega}}$, and some function $f$ witnesses $\varphi(m, z)$. By replacement, union, and comprehension we can extend $f$ to include the ordered pair $\left\langle n, \bigcup \operatorname{Rep}_{\psi} f(m)\right\rangle$; then it witnesses $\varphi\left(n, \bigcup \operatorname{Rep}_{\psi} f(m)\right)$, a contradiction.

Hence for any $n \bar{\epsilon} \bar{\omega}$ we can write $F(n)$ for the unique $y$ such that $\varphi(n, y)$.

By replacement, let $X:=\operatorname{Rep}_{\varphi} \overline{\boldsymbol{\omega}}$ (see Definition 3.11). Thus

$$
(\forall y)(y \bar{\epsilon} X \Leftrightarrow(\exists a \bar{\epsilon} \bar{\omega}) F(a)=y) .
$$

For any $v \bar{\epsilon} \bigcup X$, there exists $n \bar{\epsilon} \overline{\boldsymbol{\omega}}$ such that $v \bar{\epsilon} F(n)$.

Let $w$ be such that $\psi(v, w)$ holds. If $a \epsilon_{b} v$, then $a, b \bar{\epsilon} w$, so

$$
a, b \bar{\epsilon} \bigcup \operatorname{Rep}_{\psi} F(n)=F(n+1) .
$$

Thus $\bigcup X$ has the desired closure property, and $x \bar{\epsilon} \bigcup X$ since $x \bar{\epsilon} F(0)$.

Corollary 3.29 For any $x$ there is a $\overline{\mathrm{C}}$-minimal closed multiset containing $x$.

Proof Let $\varphi(y)$ state that $y$ is closed and $x \bar{\epsilon} y$; then there exists $y$ such that $\varphi(y)$ holds. Hence take the intersection of all multisets satisfying $\varphi$. 
Lemma 3.30 We have

$$
(\forall x, y)\left(x \bar{\subset} y \Leftrightarrow(\forall a \bar{\epsilon} x)\left(a \bar{\epsilon} y \wedge \frac{x}{a} \bar{\subset} \frac{y}{a}\right)\right) .
$$

Moreover, $(\forall x, y)(\varphi(x, y) \Rightarrow x \bar{\subset} y)$ for any formula $\varphi(x, y)$ such that

$$
(\forall x, y)\left(\varphi(x, y) \Rightarrow(\forall a \bar{\epsilon} x)\left(a \bar{\epsilon} y \wedge \varphi\left(\frac{x}{a}, \frac{y}{a}\right)\right)\right) .
$$

Proof If $x=y$ the first claim is trivial, so we assume otherwise. If $R$ witnesses $x \bar{\subset} y$, then all members of $x$ are members of $y$ and $R$ itself witnesses $\frac{x}{a} \bar{\subset} \frac{y}{a}$.

Conversely, suppose

$$
(\forall a \bar{\epsilon} x)\left(a \bar{\epsilon} y \wedge \frac{x}{a} \bar{\subset} \frac{y}{a}\right) .
$$

Let $X, Y$ be closed, and let $x \bar{\epsilon} X, y \bar{\epsilon} Y$. Define a relation $R$ as follows:

$$
R:=\left\{\langle v, w\rangle \otimes a:(\forall b \bar{\epsilon} v)\left(b \bar{\epsilon} w \wedge \frac{v}{b} \bar{c} \frac{w}{b}\right) \wedge\langle v, w\rangle \epsilon_{a} X \times Y\right\} .
$$

By the proven direction of the claim, we have

$$
(\forall v \bar{\epsilon} X)(\forall w \bar{\epsilon} Y)(v \bar{\subset} w \Rightarrow\langle v, w\rangle \bar{\epsilon} R) .
$$

From this and the definition of $R$, we have

$$
(\forall v, w)\left(\langle v, w\rangle \bar{\epsilon} R \Rightarrow(\forall b \bar{\epsilon} v)\left(b \bar{\epsilon} w \wedge\left\langle\frac{v}{b}, \frac{w}{b}\right\rangle \bar{\epsilon} R\right)\right) .
$$

But $\langle x, y\rangle \bar{\epsilon} R$ by the hypothesis, so $R$ witnesses $x \bar{\subset} y$ and the converse holds.

Now suppose that $\varphi(x, y)$ is a formula such that

$$
(\forall x, y)\left(\varphi(x, y) \Rightarrow(\forall a \bar{\epsilon} x)\left(a \bar{\epsilon} y \wedge \varphi\left(\frac{x}{a}, \frac{y}{a}\right)\right)\right)
$$

and that $\varphi(x, y)$ holds for some particular pair $x, y$. Again let $X, Y$ be closed multisets containing $x, y$, respectively, and define a relation $R$ by

$$
R:=\left\{\langle v, w\rangle \otimes a:(\forall b \bar{\epsilon} v)\left(b \bar{\epsilon} w \wedge \varphi\left(\frac{v}{b}, \frac{w}{b}\right)\right) \wedge\langle v, w\rangle \epsilon_{a} X \otimes Y\right\} .
$$

Then the same argument as above shows that $R$ witnesses $x \bar{\subset} y$.

3.5 Transitive closures There are two obvious candidates for the definition of transitive multisets:

$$
(\forall x \bar{\epsilon} a)(\forall y \bar{\epsilon} x) y \bar{\epsilon} a \quad \text { or } \quad(\forall x \bar{\epsilon} a) x \bar{\subset} a .
$$

The second trivially implies the first, but the converse is false: consider $X:=\{\bar{\emptyset} \otimes\{\bar{\emptyset} \otimes \bar{\emptyset}\}\}$ and $Y:=\{X \otimes \bar{\emptyset},\{\bar{\emptyset} \otimes \bar{\emptyset}\} \otimes \bar{\emptyset}, \bar{\emptyset} \otimes \bar{\emptyset}\} ;$ then the second condition fails for $Y$. Here we adopt the stronger condition.

Definition 3.31 A multiset $a$ is transitive if $(\forall x \bar{\epsilon} a) x \bar{C} a$.

Remark 3.32 For $X$ and $Y$ defined in the previous paragraph, $X$ is transitive but not closed while $Y$ is closed but not transitive.

Lemma 3.33 Let $\varphi(x)$ be a formula with one free variable such that $(\exists x) \varphi(x)$, and $\varphi(x)$ only holds for transitive multisets. Then $\bigcap_{\varphi} x$ is transitive.

Proof Let $a \bar{\epsilon} \bigcap_{\varphi} x$, and suppose that $\varphi(x)$ holds; then $a \bar{\epsilon} x$ since $\bigcap_{\varphi} x \bar{\subset} x$. By transitivity $a \bar{\subset} x$ for all such $x$, so $a \bar{\subset} \bigcap_{\varphi} x$ by definition. 
Remark 3.34 Ordinals are transitive: if $\alpha \in O N$ and $x \bar{\epsilon} \alpha$, then for any $y \bar{\epsilon} x$ we have $y \bar{\epsilon} \alpha$ and $\frac{x}{y}=\bar{\emptyset} \bar{\subset} \frac{\alpha}{y}$; hence $x \bar{\subset} \alpha$.

Lemma 3.35 For any multiset $x$, there exists a $\overline{\mathrm{C}}$-minimal transitive multiset $T C(x)$ such that $x \bar{\subset} T C(x)$.

Proof Let $x$ be any multiset. We can define a function class $\varphi$ on $\bar{\omega}$ (see Lemma 3.28) such that $\varphi(0)=x$ and $\varphi(\alpha+1)=\bigcup \varphi(\alpha)$ for all $\alpha \in \bar{\omega}$.

Hence by replacement there exists $v:=\{b \otimes \bar{\emptyset}:(\exists a \bar{\epsilon} \bar{\omega}) \varphi(a, b)\}$.

Let $w:=\bigcup v$; then $x \bar{\subset} w$ since $x \bar{\epsilon} v$. Given $e \bar{\epsilon} w$, by Lemma 3.12, $(\exists \alpha) e \bar{\epsilon} \varphi(\alpha)$. Then $\bigcup \varphi(\alpha)=\varphi(\alpha+1) \bar{\epsilon} v$, so $\varphi(\alpha) \bar{\subset} w$. But $e \bar{\subset} \varphi(\alpha)$, so $e \bar{\subset} w$.

Thus there is a transitive $w$ such that $x \bar{\subset} w$, so let $T C(x)$ be the intersection of all such $w$. By Lemma 3.3, TC $(x)$ is transitive, and by definition of intersection $x \bar{\subset} T C(x)$ and $T C(x)$ is $\overline{\mathrm{C}}$-minimal.

Corollary 3.36 We have the following:

$$
\begin{aligned}
& (\forall x, y)(x \bar{\epsilon} T C(y) \Rightarrow T C(x) \bar{\subset} T C(y)), \\
& (\forall x, y)(x \bar{\epsilon} T C(y) \Leftrightarrow(x \bar{\epsilon} y \vee(\exists a \bar{\epsilon} y) x \bar{\epsilon} T C(a))) .
\end{aligned}
$$

Proof If $x \bar{\epsilon} T C(y)$, then $x \bar{\subset} T C(y)$, so by definition $T C(x) \bar{\subset} T C(y)$ and the left-to-right direction of the second claim follows trivially.

Conversely, suppose $x \notin y$ and $(\forall a \bar{\epsilon} y) x \notin T C(a)$. By replacement, comprehension, and union let

$$
v:=y \cup \bigcup\left\{T C(a) \otimes b: a \epsilon_{b} y\right\} .
$$

If $w \bar{\epsilon} v$, then $w \neq x$. Either $w \bar{\epsilon} y$ so $w \bar{\subset} T C(w) \bar{\subset} v$, or $w \bar{\epsilon} T C(a)$ for some $a \bar{\epsilon} y$ so $w \bar{\subset} T C(a) \bar{\subset} v$. Hence $v$ is transitive and $T C(y) \bar{\subset} v$, so $x \notin T C(y)$.

We propose the following basic system, leaving foundation for later discussion.

Definition 3.37 The theory MS consists of the following axioms and schemas: unique multiplicity, extensionality, comprehension, pairing, subset, union, replacement, power set, and infinity.

3.6 The collection of sets In this subsection we work in a model of MS.

Definition 3.38 For any multiset $x$, if there exists $c$ such that

$$
(\forall y)(y \bar{\epsilon} c \Leftrightarrow y \bar{\epsilon} x) \wedge(\forall y \bar{\epsilon} c) \frac{c}{y}=\bar{\emptyset},
$$

we say that $c=\operatorname{core}(x)$. Write $x \in$ Core for $(\forall y \bar{\epsilon} x) \frac{x}{y}=\bar{\emptyset}$.

Remark 3.39 The axioms of MS might not guarantee that core $(x)$ exists for all $x$, but core $(x)$ is unique by extensionality if it exists.

It trivially follows that the core of $\operatorname{core}(x)$ is itself, and two multisets have the same core if and only if they have the same members. Note that if $\alpha \in O N$, then $\operatorname{core}(\alpha)=\alpha$ by definition.

Definition 3.40 We say that $x \in$ Set if $x \in \operatorname{Core} \wedge(\forall y \bar{\epsilon} T C(x)) y \in$ Core. 
It immediately follows that $(\forall x)(x \in$ Set $\Leftrightarrow x \in$ Core $\wedge(\forall y \bar{\epsilon} x) y \in$ Set $)$.

Consider the following interpretation of the language of set theory: $=$ is the identity relation, $\in$ is interpreted as $\bar{\epsilon}$, whereas $\exists$ and $\forall$ are relative to Set. The settheoretic inclusion relation under this interpretation coincides with $\bar{C}$, that is,

$$
(\forall x, y \in \operatorname{Set})(x \bar{\subset} y \Leftrightarrow(\forall a \bar{\epsilon} x) a \bar{\epsilon} y) .
$$

Theorem 3.41 If MS is consistent, so is ZF.

Proof The given interpretation turns Set into a model of ZF minus foundation. For infinity we have $\overline{\boldsymbol{\omega}} \in$ Set, while for any other axiom the multiset given by its multiset counterpart is in Set as long as the parameters are in Set.

Remark 3.42 Note that Definition 3.23 ensures that $(\forall \alpha \in O N) \alpha \in$ Set.

Remark 3.43 Up to this point, since we have avoided manipulation of multiplicities, note that any model of ZF set theory provides a model for MS by interpreting $x \in_{a} y \Leftrightarrow d f x \in y \wedge a=\emptyset$. Hence the consistency strength of MS is trivially the same as ZF.

3.7 Multiplicity replacement By adding different axioms to handle multiplicities, we can extend MS to implement different systems of multisets of various strengths. For example, consider the following theory.

\section{Axiom 3.7.1 (Axiom of finite multiplicities) We have}

$$
(\forall x, y, a)\left(x \epsilon_{a} y \Rightarrow a \bar{\epsilon} \bar{\omega}\right) \wedge(\forall x)(\forall a \bar{\epsilon} \bar{\omega})(\exists y) y=\{x \otimes a\} .
$$

Definition 3.44 The theory $\mathrm{MS}_{\bar{\omega}}$ consists of all MS axioms, plus the axiom of finite multiplicities.

The theory $\mathrm{MS}_{\bar{\omega}}$ describes a system of multisets with finite multiplicities, essentially equivalent to the theory MST in [2]. The one major difference is that in MST the multiplicity 0 means nonmembership, that is, the multiplicity $n$ in $\mathrm{MS}_{\bar{\omega}}$ corresponds to the multiplicity $n+1$ in MST.

To define a theory where multiplicities are ZF cardinals like MSTC in [5], note that Set is a model of ZF. Thus we would only need to add the following axioms to MS (where Card is the class of set-theoretic cardinals defined in Set).

Axiom Schema 3.7.2 For any $\varphi$ with two free variables and possibly parameters

$$
(\forall x)((\forall a \bar{\epsilon} x)(\exists ! b \bar{\epsilon} \operatorname{Card}) \varphi(a, b) \Rightarrow(\exists y) y=\{a \otimes b: a \bar{\epsilon} x \wedge \varphi(a, b)\}) .
$$

Axiom 3.7.3 We have $(\forall x, y, a)\left(x \in_{a} y \Rightarrow a \bar{\epsilon}\right.$ Card $)$.

Remark 3.45 As long as our axiom is strong enough to replace any multiplicity by $\bar{\emptyset}$, two multiset ordinals will have a bijection in the universe of multisets if and only if they have a bijection in the class Set; thus the alephs in the multiset model will be the same as the set-theoretic alephs in the ZF interpretation of Set.

The strongest axiom to manipulate multiplicities is given below.

Axiom Schema 3.7.4 (Axiom schema of multiplicity replacement) We have

$$
(\forall x)((\forall a \bar{\epsilon} x)(\exists ! b) \varphi(a, b) \Rightarrow(\exists y) y=\{a \otimes b: a \bar{\epsilon} x \wedge \varphi(a, b)\})
$$

for any formula $\varphi$ with two free variables and possibly parameters. 
With this axiom, our multiset theory can no longer accept models of set theory since set-theoretic extensionality is provably false: for example, $\{\bar{\varnothing} \otimes \bar{\emptyset}\}$ and $\{\bar{\varnothing} \otimes\{\bar{\varnothing} \otimes \bar{\emptyset}\}\}$ are distinct multisets with exactly the same members. In the same way, it cannot accept models of any traditional multiset theory where multiplicities are integers or cardinals, since multiplicity replacement can create multiplicities not allowed by those theories.

Definition 3.46 The theory MSR consists of MS plus multiplicity replacement.

Lemma 3.47 We have (MSR) $(\forall x)(\forall a \in \bigcup x)\left(\frac{\bigcup x}{a}=\bigcup\left\{\frac{y}{a} \otimes b: y \epsilon_{b} x \wedge\right.\right.$ $a \bar{\epsilon} y\})$.

Proof By multiplicity replacement let

$$
A:=\left\{v \otimes w: v \bar{\epsilon} \bigcup x \wedge w=\bigcup\left\{\frac{y}{v} \otimes b: y \epsilon_{b} x \wedge v \bar{\epsilon} y\right\}\right\} .
$$

Let $y \bar{\epsilon} x$. If $v \bar{\epsilon} y$, then $v \bar{\epsilon} \bigcup x$, so $v \bar{\epsilon} A$ and $\frac{y}{v} \bar{C} \frac{A}{v}$ by definition of $A$. By Lemma 3.30, $y \bar{\subset} A$; thus $\bigcup x \bar{\subset} A$, so $(\forall a \bar{\epsilon} \bigcup x) \frac{\bigcup x}{a} \bar{\subset} \frac{A}{a}$.

Conversely, if $a \bar{\epsilon} y \bar{\epsilon} x$, then $y \bar{\subset} \cup x$, so $\frac{y}{a} \bar{\subset} \frac{\cup x}{a}$. Hence $\frac{A}{a} \bar{\subset} \frac{\bigcup x}{a}$, and the claim follows by antisymmetry of $\bar{C}$.

3.8 Well-founded multisets Start with a model of MSR.

Definition 3.48 Say that $x$ is well founded if every submultiset $A$ of the $\overline{\mathrm{C}}$ minimal multiset containing $x$ (see Corollary 3.29) has a minimal member $y$ such that

$$
(\forall z \bar{\epsilon} y)\left(z \notin A \wedge \frac{y}{z} \bar{\notin} A\right) .
$$

Alternatively we can define the class $W F$ of well-founded multisets by an analogue of the von Neumann hierarchy, where $V_{\bar{\emptyset}}:=\bar{\emptyset}$,

$$
V_{\alpha+1}:=\left\{x \otimes \bar{\emptyset}:(\forall y, a)\left(y \epsilon_{a} x \Rightarrow\left(y \bar{\epsilon} V_{\alpha} \wedge a \bar{\epsilon} V_{\alpha}\right)\right)\right\},
$$

and $V_{\lambda}:=\bigcup\left\{V_{\alpha} \otimes \bar{\emptyset}: \alpha<\lambda\right\}$ for limit $\lambda$.

A simple induction shows that the $V_{\alpha}$ are all closed and nested and that $W F$ is precisely the class of well-founded multisets. We can then define the rank of $x \in W F$ as the minimal $\alpha$ such that $x \bar{\epsilon} V_{\alpha}$, and thus

$$
\operatorname{rank} x:=\max \left\{\sup _{y \bar{\epsilon} x} \operatorname{rank} y+1, \sup _{y \bar{\epsilon} x} \operatorname{rank} \frac{x}{y}+1\right\} .
$$

Lemma 3.49 We have $(\forall x)\left((\forall y \bar{\epsilon} x)\left(y \in W F \wedge \frac{x}{y} \in W F\right) \Rightarrow x \in W F\right)$.

Proof Let $\alpha:=\max \left\{\sup _{y \bar{\epsilon} x} \operatorname{rank} y+1, \sup _{y \bar{\epsilon} x} \operatorname{rank} \frac{x}{y}+1\right\}$; then $x \bar{\epsilon} V_{\alpha+1}$.

Lemma 3.50 We have $(\forall x \in W F)(\forall y \bar{C} x) y \in W F$.

Proof If there exist $x$ and $y$ contradicting the claim, let $x$ be of minimal rank.

Lemma 3.51 We have $(\forall \alpha \in O N) \alpha \in W F$.

Proof Since ordinals are closed, it is easy to show that $\alpha^{+}$is the $\overline{\mathrm{C}}$-minimal closed multiset that has $\alpha$ as a member. Suppose $A \overline{\mathcal{C}} \alpha^{+}$, and let $y \bar{\epsilon} A$ be the $\bar{\epsilon}$-minimal; then $y$ satisfies Definition 3.48 straightforwardly.

Axiom 3.8.1 (Axiom of foundation) $\quad$ We have $(\forall v) v \in W F$. 
Theorem 3.52 WF is a model of MSR plus foundation.

Proof Extensionality holds since $W F$ is closed downwards. Comprehension, pairing, power set, and multiplicity replacement all hold by Lemma 3.49. Union holds by an induction on rank, using the recursive relation in Lemma 3.47. Replacement follows from union and Lemma 3.49. Infinity holds since $\bar{\omega}$ is well founded by Lemma 3.51. Foundation holds since the cumulative hierarchy defined relative to $W F$ is exactly the same as the cumulative hierarchy that forms $W F$.

Lemma 3.53 (MSR) For any multiset $x$ there is a transitive closed multiset $y$ such that $x \bar{\epsilon} y$ (see Corollary 3.29 and Lemma 3.35).

Proof Let $a$ be closed such that $\{x \otimes \bar{\emptyset}\} \bar{\epsilon} a$, and let $b=\bigcup a$; then $x \bar{\epsilon} b$.

If $v \bar{\epsilon} b$, then $v \bar{\epsilon} w$ for some $w \bar{\epsilon} a$, so $v \bar{\epsilon} a$. Thus $v \bar{\subset} b$; that is, $b$ is transitive.

Also $\frac{w}{v} \bar{\epsilon} a$ whenever $v \bar{\epsilon} w \bar{\epsilon} a$; thus

$$
\frac{b}{v}=\bigcup\left\{\frac{w}{v} \otimes \bar{\emptyset}: v \bar{\epsilon} w \bar{\epsilon} a\right\} \bar{\subset} b .
$$

Let $c$ be the smallest closed multiset such that $b \bar{\epsilon} c$. Then an easy induction on the minimal property of $c$ shows that for all $w \bar{\epsilon} c$,

$$
w \bar{\subset} b \wedge(\forall v \bar{\epsilon} w) \frac{w}{v} \bar{\subset} b .
$$

By multiplicity replacement let $y:=\{v \otimes b: v \bar{\epsilon} c\}$; then $y$ is also closed and obviously $x \overline{\boldsymbol{\epsilon}} y$. Let $w \bar{\epsilon} y$; then $w \bar{\epsilon} c$, so $(\forall v \bar{\epsilon} w) \frac{w}{v} \bar{\subset} b=\frac{y}{v}$. Hence $w \bar{\subset} y$, that is, $y$ is transitive, as required.

Remark 3.54 Any model of Zermelo (not ZF) set theory that refutes transitive containment (such as that given in Mathias [10]) readily provides a model of MS minus replacement in which some multiset is not contained in any transitive multiset.

\section{A Model for the Theory}

We prove the consistency of MSR relative to ZF, starting with a model $V$ of ZF.

It is possible to construct a well-founded model for our multiset theory from $V$ by interpreting $x \in_{a} \quad y$ in the language of multisets as $\langle x, a\rangle \in y$ within a recursively defined subclass of $V$. However, here we will construct a graph-based model, which will give us the consistency of MSR with antifoundation. Furthermore, in any wellfounded model of multiset theory the inclusion relation is necessarily antisymmetric (by induction on the membership relation), whereas our graph-based model will also prove the independence of the axiom of subset from the rest of MSR.

4.1 Accessible pointed hypergraphs We will only consider directed 3-uniform hypergraphs; our model will be a definable class of these hypergraphs. A hypergraph $H$ is thus a set of ordered triples $\langle x, y, z\rangle$ (i.e., its edges).

Remark 4.1 We sometimes write $H(x, y, z)$ as shorthand for $\langle x, y, z\rangle \in H$.

Definition 4.2 Let $H$ be a hypergraph, and let $x$ be any set.

Let $H^{-1} x:=\{y:(\exists z)\langle x, y, z\rangle \in H\}$ and $H^{-2} x:=\{z:(\exists y)\langle x, y, z\rangle \in H\}$.

Let $H^{-1}:=\{y:(\exists x, z)\langle x, y, z\rangle \in H\}$ and $H^{-2}:=\{z:(\exists x, y)\langle x, y, z\rangle \in H\}$. 
Definition 4.3 A pointed hypergraph $[H, h]$ is an ordered pair $\langle H, h\rangle$ where $H$ is a hypergraph and $h$ a set (often a vertex of $H$ ) which we call the point of $[H, h]$. Say that $[G, h] \cong[H, h]$ if there is an isomorphism between $G$ and $H$ that takes $g$ to $h$.

Definition 4.4 Write $\operatorname{Dom}[H, h]$ for the set of vertices of $H$, plus $h$.

Definition 4.5 A finite directed path from $x_{1}$ to $x_{n}$ in $[H, h]$ is a finite sequence $x_{1} \cdots x_{n}$ in $\operatorname{Dom}[H, h]$, where $x_{i+1} \in H^{-1} x_{i}$ or $x_{i+1} \in H^{-2} x_{i}$.

Definition 4.6 We have $x_{H}:=\bigcap\left\{X: x \in X \wedge(\forall y \in X)\left(H^{-1} y \subset X \wedge H^{-2} \times\right.\right.$ $y \subset X)\}$;

$x_{H}$ contains $x$ and all vertices of $H$ accessible from $x$ by a finite directed path.

Definition 4.7 Let $H_{x}$ be the restriction of $H$ to $x_{H}$. It is the smallest subgraph of $H$ containing $x$ and closed under outward edges.

Definition 4.8 The pointed hypergraph $[H, h]$ is accessible if $\operatorname{Dom}[H, h]=h_{H}$. Equivalently, for any $x \in \operatorname{Dom}[H, h]$ there is a finite directed path in $[H, h]$ from $h$ to $x$.

Remark 4.9 For any hypergraph $H$ and any $x,\left[H_{x}, x\right]$ is accessible.

4.2 Bisimulations and extensionality To enforce extensionality, we borrow the concept of a bisimulation from computer science. Our definition of multiset bisimulation was adapted from Aczel [1] and is very close to what D'Agostino and Visser use in [6] and [7], except that they have a fixed implementation of multiplicities (which are positive cardinals). In fact our model, when restricted to cardinal multiplicities, describes essentially the same multiset universe as in [6], though the particular constructions are different: while the latter followed a parallel course to Aczel's approach in [1], ours is generalized from the graph-based models of set theory in Hinnion [8] and Holmes [9].

Definition 4.10 Call a relation $\sim \subset \operatorname{Dom}[G, g] \times \operatorname{Dom}[H, h]$ a bisimulation between $[G, g]$ and $[H, h]$ if for any $a \sim x$ we have

$$
\begin{aligned}
& (\forall b, c)(G(a, b, c) \Rightarrow(\exists y, z)(H(x, y, z) \wedge b \sim y \wedge c \sim z)) \\
& \quad \wedge(\forall y, z)(H(x, y, z) \Rightarrow(\exists b, c)(G(a, b, c) \wedge b \sim y \wedge c \sim z)) .
\end{aligned}
$$

If $[G, g]=[H, h]$, we say that $\sim$ is a bisimulation on $[H, h]$.

Lemma 4.11 Let $\sim$ be a bisimulation between $[G, g]$ and $[H, h]$; then we have the following.

1. The relation $\simeq:=\{\langle x, y\rangle: y \sim x\}$ is a bisimulation between $[H, h]$ and $[G, g]$.

2. If $\simeq$ is a bisimulation between $[H, h]$ and $[Q, q]$, then $x \approx d \Leftrightarrow \Leftrightarrow_{d f}(\exists a)(x \sim$ $a \wedge a \simeq d)$ is a bisimulation between $[G, g]$ and $[Q, q]$.

3. The restriction of $\sim$ to $\operatorname{Dom}\left[G_{x}, x\right] \times \operatorname{Dom}\left[H_{y}, y\right]$ is a bisimulation between $\left[G_{x}, x\right]$ and $\left[H_{y}, y\right]$.

4. Any bisimulation between $\left[G_{x}, x\right]$ and $\left[H_{y}, y\right]$ is a bisimulation between $[G, g]$ and $[H, h]$.

5. If $[G, g],[H, h]$ are accessible and $g \sim h$, then

$$
(\forall y \in \operatorname{Dom}[H, h])(\exists x) x \sim y \wedge(\forall x \in \operatorname{Dom}[G, g])(\exists y) x \sim y .
$$




\section{Proof}

1. The result is immediate from the definition of bisimulation.

2. Let $x \sim a \simeq d$ and $G(x, y, z)$. There are $b, c \in \operatorname{Dom}[H, h]$ such that $y \sim b$, $z \sim c$, and $H(a, b, c)$. Hence there are $e, f \in[Q, q]$ such that $b \simeq e, c \simeq f$, and $Q(d, e, f)$, but then we have $y \approx e$ and $z \approx f$.

The other direction is similar, so $\approx$ is a bisimulation.

3. If $a \sim d$ where $a \in \operatorname{Dom}\left[G_{x}, x\right], d \in \operatorname{Dom}\left[H_{y}, y\right]$, and $G_{x}(a, b, c)$, then clearly $G(a, b, c)$. Hence there are $e, f \in \operatorname{Dom}[H, h]$ such that $b \sim e$, $c \sim f$, and $H(d, e, f)$. But then $H_{y}(d, e, f)$ by definition of $\left[H_{y}, y\right]$.

The other direction is similar.

4. If $a \sim d$ and $G(a, b, c)$, then $G_{x}(a, b, c)$ by definition of $\left[G_{x}, x\right]$. Thus there are $e, f \in \operatorname{Dom}\left[H_{y}, y\right]$ such that $b \sim e, d \sim f$, and $G_{y}(d, e, f)$. Hence $G(d, e, f)$, and the other direction is similar.

5. Let $y \in \operatorname{Dom}[H, h]$. There exists a finite sequence $y_{1} \cdots y_{n}$ in $\operatorname{Dom}[H, h]$ where $y_{1}=h, y_{n}=y$, and $y_{i+1} \in H^{-1} y_{i} \cup H^{-2} y_{i}$ for all $i$. We show by induction on $n$ that there is a sequence $x_{1} \cdots x_{n}$ in $\operatorname{Dom}[G, g]$ such that $x_{i} \sim y_{i}$ and $x_{i+1} \in G^{-1} x_{i} \cup G^{-2} x_{i}$ for all $i$. The case $n=1$ is trivial.

If $n>1$, by the induction hypothesis we have a sequence $x_{1} \cdots x_{n-1}$ such that $x_{i} \sim y_{i}$ and $x_{i+1} \in G^{-1} x_{i} \cup G^{-2} x_{i}$ for all $i$. Now $y_{n} \in H^{-1} y_{n-1} \cup$ $H^{-2} y_{n-1}$ and $x_{n-1} \sim y_{n}-1$, so there exists $x_{n} \in H^{-1} x_{n-1} \cup H^{-2} x_{n-1}$ such that $x_{n} \sim y_{n}$ since $\sim$ is a bisimulation.

We proceed similarly for the other direction.

Definition 4.12 Say that $[H, h]$ is extensional if the only nonempty bisimulation on $[H, h]$ is (contained in) the identity.

Lemma 4.13 Let $[H, h]$ be extensional; then $\left[H_{x}, x\right]$ is extensional for any $x \in \operatorname{Dom}[H, h]$. Furthermore, if $\left[H_{x}, x\right] \cong\left[H_{y}, y\right]$ for $x, y \in \operatorname{Dom}[H, h]$, then $x=y$.

Proof The first claim follows trivially from Lemma 4.11(3) and (4), whereas the second claim follows from (4) of the same lemma.

4.3 The domain of our model We will define a canonical member for each equivalence class. The collection of those forms a definable class of hypergraphs, which we will denote by $\mathcal{M}$.

Lemma 4.14 (Quotient lemma) For any pointed hypergraph $[H, h]$, there exists an extensional pointed hypergraph $[Q, q]$ and a surjective quotient map $\pi$ from $\operatorname{Dom}[H, h]$ to $\operatorname{Dom}[Q, q]$ such that $q=\pi(h)$ and

$$
(\forall a, b, c)(Q(a, b, c) \Leftrightarrow(\exists x, y, z)(a=\pi(x) \wedge b=\pi(y) \wedge c=\pi(z)))
$$

and such that the relation $\pi(x)=y$ is a bisimulation between $[H, h]$ and $[Q, q]$.

We call $[Q, q]$ the extensional quotient of $[H, h]$. Furthermore, we have the following.

1. The extensional quotient is unique up to isomorphism.

2. We have $\operatorname{Dom}\left[Q_{\pi(x)}, \pi(x)\right]=\left\{\pi(y): y \in \operatorname{Dom}\left[H_{x}, x\right]\right\}$. In particular, if $[H, h]$ is accessible, then so is $[Q, q]$ (by taking $x=h$ ).

3. The extensional quotient of $\left[H_{x}, x\right]$ is (isomorphic to) $\left[Q_{\pi(x)}, \pi(x)\right]$.

4. If $\left[H_{x}, x\right]$ is extensional, then $\left[Q_{\pi(x)}, \pi(x)\right]$ is isomorphic to $\left[H_{x}, x\right]$. 
Proof If $\sim \subset \operatorname{Dom}[H, h]^{2}$, define $\sim^{+}$by

$$
\begin{aligned}
& a \sim^{+} x \Leftrightarrow_{d f}(\forall b, c)(H(a, b, c) \Rightarrow(\exists y, z)(H(x, y, z) \wedge b \sim y \wedge c \sim z)) \\
& \wedge(\forall y, z)(H(x, y, z) \Rightarrow(\exists b, c)(H(a, b, c) \wedge b \sim y \wedge c \sim z)) .
\end{aligned}
$$

Clearly $\sim_{1} \subset \sim_{2}$ implies $\sim_{1}^{+} \subset \sim_{2}^{+}$and $\sim$ is a bisimulation if and only if $\sim \subset \sim^{+}$. Let $\approx \subset \operatorname{Dom}[H, h]^{2}$ be the union of all bisimulations on $[H, h]$, that is,

$$
x \approx y \Leftrightarrow d f(\exists \sim)\left(\sim \subset \sim^{+} \wedge x \sim y\right) .
$$

The identity on $\operatorname{Dom}[H, h]$ is a bisimulation, so $\approx$ is reflexive. By Lemma 4.11 the relations $\{\langle y, x\rangle: x \approx y\}$ and $\{\langle x, z\rangle:(\exists y) x \approx y \approx z\}$ are also bisimulations, so $\approx$ is symmetric and transitive. The set of relations on $\operatorname{Dom}[H, h]$ ordered by $\subset$ forms a complete lattice, and the operation $\sim \mapsto \sim^{+}$is monotonic. Hence by the Knaster-Tarski theorem (see, e.g., Tarski [11]) $\approx$ is the same as $\approx^{+}$.

Let $\operatorname{Dom}[Q, q]$ be the set of equivalence classes of $\approx$, let $q$ be the equivalence class of $h$, and let $\pi: \operatorname{Dom}[H, h] \rightarrow \operatorname{Dom}[Q, q]$ be the corresponding quotient map.

Define the relation $Q$ on $\operatorname{Dom}[Q, q]$ by

$$
Q(a, b, c) \Leftrightarrow_{d f}(\exists x, y, z)(H(x, y, z) \wedge \pi(x)=a \wedge \pi(y)=b \wedge \pi(z)=c) .
$$

We show that any bisimulation $\sim Q$ on $[Q, q]$ must be the identity.

Define $x \sim_{H} y \Leftrightarrow_{d f} \pi(x) \sim_{Q} \pi(y)$; then it is straightforward to check that $\sim_{H}$ is a bisimulation. This means that $\sim_{H} \subset \approx$, so $\pi(x)=\pi(y)$ for any $x \sim_{H} y$, and thus $\sim Q$ is the identity. We have shown that $[Q, q]$ is extensional.

If $\pi(x)=a$ and $Q(a, b, c)$, there are $d, e, f$ such that $\pi(d)=a, \pi(e)=b$, $\pi(f)=c$, and $H(d, e, f)$. Then $x \approx d$, so there are $y \approx e, z \approx f$ such that $H(x, y, z)$. But then $\pi(y)=b$ and $\pi(z)=c$.

On the other hand, if $H(x, y, z)$, then $Q(\pi(x), \pi(y), \pi(z))$. Thus the relation $\pi(x)=y$ is a bisimulation between $[H, h]$ and $[Q, q]$.

Now we turn to prove the further claims in the lemma.

1. Define $x \sim y \Leftrightarrow d f \pi(x)=\pi(y)$; then $\sim$ is a bisimulation on $[H, h]$ by Lemma 4.11(2) since the relation $\pi(x)=y$ is a bisimulation. Similarly, given a bisimulation $\approx$ on $[H, h]$ we have a bisimulation on $[Q, q]$,

$$
x \simeq y \Leftrightarrow_{d f}(\exists a, b)(x=\pi(a) \wedge a \approx b \wedge \pi(b)=y) .
$$

But $[Q, q]$ is extensional, so $\simeq$ is the identity, and thus $\approx \subset \sim$. Hence $\sim$ is the union of all bisimulations on $[H, h]$, which is unique.

2. $\left[H_{x}, x\right]$ and $\left[Q_{\pi(x)}, \pi(x)\right]$ are accessible, and by Lemma 4.11(3) the relation $\pi(x)=y$ is a bisimulation between them. Hence by part (5) of the same lemma $\operatorname{Dom}\left[Q_{\pi(x)}, \pi(x)\right]=\pi$ “ $\operatorname{Dom}\left[H_{x}, x\right]$.

3. Let $G$ be the restriction of $Q$ to $\pi$ " $\operatorname{Dom}\left[H_{x}, x\right]$, and let $\approx$ be the union of all bisimulations on $[H, h]$. By Lemma 4.11 the restriction of $\approx$ to $\operatorname{Dom}\left[H_{x}, x\right]^{2}$ is the union of all bisimulations on $\left[H_{x}, x\right]$, so the extensional quotient of $\left[H_{x}, x\right]$ is isomorphic to $[G, \pi(x)]$ by mapping the equivalence class of any vertex $a$ in $\operatorname{Dom}\left[H_{x}, x\right]$ to the equivalence class of $a$ in $\operatorname{Dom}[H, h]$.

By part (2) of this lemma $[G, \pi(x)]$ is the same as $\left[Q_{\pi(x)}, \pi(x)\right]$.

4. If $\left[H_{x}, x\right]$ is extensional, the restriction of any bisimulation on $[H, h]$ to $\operatorname{Dom}\left[H_{x}, x\right]=x_{H}$ is the identity. Hence $\pi: \operatorname{Dom}\left[H_{x}, x\right] \leftrightarrow \operatorname{Dom}\left[Q_{\pi(x)}, x\right]$ is a bijection by construction. 
Suppose $a, b, c \in \operatorname{Dom}\left[H_{x}, x\right]$ and $Q(\pi(a), \pi(b), \pi(c))$; then there are $d, e \in \operatorname{Dom}[H, h]$ such that $\pi(d)=\pi(b), \pi(e)=\pi(c)$, and $H(a, d, e)$. But then $d, e \in \operatorname{Dom}\left[H_{x}, x\right]$, so $d=b$ and $e=c$, that is, $H(a, b, c)$.

Conversely, we already know that $H(a, b, c) \Rightarrow Q(\pi(a), \pi(b), \pi(c))$; hence $\left[Q_{\pi(x)}, \pi(x)\right] \cong\left[H_{x}, x\right]$.

Definition 4.15 The hypergraph $[Q, q]$ constructed in the quotient lemma provides a canonical example of an extensional quotient of $[H, h]$. From now on we simply refer to it as the extensional quotient of $[H, h]$.

Definition 4.16 Say that $[G, g]$ and $[H, h]$ are similar if their extensional quotients are isomorphic, and write $[G, g] \equiv[H, h]$.

Remark 4.17 It is immediate from the definition above and the quotient lemma that if $[G, g]$ and $[H, h]$ are extensional, then $[G, g] \equiv[H, h] \Leftrightarrow[G, g] \cong[H, h]$.

Lemma 4.18 Let $[G, g]$ and $[H, h]$ be accessible. Then $[G, g] \equiv[H, h]$ if and only if there exists a bisimulation $\sim$ between $[G, g]$ and $[H, h]$ such that $g \sim h$.

Proof Let $\pi_{G}:[G, g] \rightarrow[P, p]$ and $\pi_{H}:[H, h] \rightarrow[Q, q]$ be the quotient maps.

If $\theta$ is an isomorphism between $[P, p]$ and $[Q, q]$, then the relation $\theta(x)=y$ is clearly a bisimulation, so $x \sim y \Leftrightarrow_{d f} \theta \pi_{G}(x)=\pi_{H}(y)$ is a bisimulation by Lemma 4.11(2). Furthermore, $\theta \pi_{G}(g)=\theta(p)=q=\pi_{H}(h)$, so $g \sim h$.

Conversely, let $\sim$ be a bisimulation between $[G, g]$ and $[H, h]$ such that $g \sim h$. Define relations $\approx$ between $\operatorname{Dom}[P, p]$ and $\operatorname{Dom}[Q, q]$ and $\simeq$ on $\operatorname{Dom}[P, p]$ by

$$
\begin{aligned}
& x \approx a \Leftrightarrow_{d f}(\exists y, b)\left(\pi_{G}(y)=x \wedge \pi_{H}(b)=a \wedge y \sim b\right), \\
& x \simeq y \Leftrightarrow_{d f}(\exists a \in \operatorname{Dom}[Q, q])(x \approx a \wedge y \approx a) .
\end{aligned}
$$

By Lemma 4.11(2) again both are bisimulations. Since $[P, p]$ is extensional, $\simeq$ is the identity, so $\approx$ is a partial function from $\operatorname{Dom}[P, p]$ to $\operatorname{Dom}[Q, q]$.

The same reasoning with $[Q, q]$ shows that $\approx$ is injective. Moreover, $p \approx q$ and the hypergraphs are accessible, so by Lemma 4.11 we know that $\approx$ is defined on the whole of $\operatorname{Dom}[P, p]$ and surjective on $\operatorname{Dom}[Q, q]$.

Thus $\approx$ is a bijection between $\operatorname{Dom}[P, p]$ and $\operatorname{Dom}[Q, q]$, but it is also a bisimulation between $[P, p]$ and $[Q, q]$, and furthermore $p \approx q$.

Corollary 4.19 For any $x, y \in \operatorname{Dom}[H, h],\left[H_{x}, x\right] \equiv\left[H_{y}, y\right]$ if and only if there is a bisimulation $\sim$ on $[H, h]$ such that $x \sim y$.

Proof The proof is immediate from the Lemma 4.18 and Lemma 4.11.

We are now ready to define the class of canonical representations for multisets.

Definition 4.20 We call $[H, h]$ a multigraph if it is accessible, extensional, and

$$
(\forall a, b, c, d \in \operatorname{Dom}[H, h])(H(a, b, c) \wedge H(a, b, d) \Rightarrow c=d) .
$$

Write $\mathcal{M}$ for the class of multigraphs.

Remark 4.21 Note that our definition of a multigraph is quite different from that of [6] and [7] despite the similarity of the eventual construction. The reason is both our different axiomatization of multiplicities and that our multigraphs are defined to be already extensional (or "collapsed" in other words). 
Lemma 4.22 Let $[H, h]$ be accessible, and for all $a, b, c, d, e \in \operatorname{Dom}[H, h]$, let

$$
\left(H(a, b, c) \wedge H(a, d, e) \wedge\left[H_{b}, b\right] \equiv\left[H_{d}, d\right]\right) \Rightarrow\left[H_{c}, c\right] \equiv\left[H_{e}, e\right] .
$$

Then the extensional quotient $[Q, q]$ of $[H, h]$ is a multigraph.

Proof Let $\pi$ be the quotient map from $[H, h]$ to $[Q, q]$. By construction $[Q, q]$ is extensional, and its accessibility comes from $[H, h]$.

If $Q(\pi(a), \pi(b), \pi(c))$ and $Q(\pi(a), \pi(b), \pi(d))$, without loss of generality assume $H(a, b, c)$. Since the relation $\pi(x)=y$ is a bisimulation between $[H, h]$ and $[Q, q]$, there exist $e, f \in \operatorname{Dom}[H, h]$ such that $\pi(e)=\pi(b), \pi(f)=\pi(d)$, and $H(a, e, f)$.

The relation $\pi(x)=\pi(y)$ is a bisimulation on $[H, h]$, so $\left[H_{e}, e\right] \equiv\left[H_{b}, b\right]$ and $\left[H_{f}, f\right] \equiv\left[H_{d}, d\right]$ by Corollary 4.19. By the hypothesis $\left[H_{f}, f\right] \equiv\left[H_{c}, c\right]$, so $\left[H_{c}, c\right] \equiv\left[H_{d}, d\right]$. By Corollary 4.19 again, there is a bisimulation $\sim$ on $[H, h]$ such that $c \sim d$, so $\pi(c)=\pi(d)$, as required.

Definition 4.23 If $[H, h],[Q, q] \in \mathcal{M}$, say, $[Q, q] \bar{\epsilon}[H, h]$ if there exists $d \in H^{-1} h$ such that $[Q, q] \cong\left[H_{d}, d\right]$.

Definition 4.24 If $[Q, q] \bar{\epsilon}[H, h]$, there is a unique edge $\langle h, n, v\rangle \in H$ such that $\left[H_{n}, n\right] \cong[Q, q]$. Let $\frac{[H, h]}{[Q, q]}:=\left[H_{v}, v\right]$.

Remark 4.25 The definitions above overload the notation of the language of multisets in an obvious manner and will be used in the following interpretation.

Given any formula $\varphi$ in the language of multiset (see Definition 3.1), form the formula $\varphi^{\mathcal{M}}$ in the language of set theory by

- restricting all universal and existential quantifiers to the class $\mathcal{M}$;

- replacing the identity relation with the bisimilarity relation $\equiv$;

- replacing the membership relation $x \in_{a} y$ with $x \bar{\epsilon} y \wedge a=\frac{y}{x}$.

Definition 4.26 Write $\mathcal{M} \models \varphi$ to say that $\varphi^{\mathcal{M}}$ is true in the starting ZF model.

We proceed to prove the axioms of MSR under the given interpretation.

\subsection{Extensionality and subset}

Lemma 4.27 (Axiom of extensionality) Two pointed hypergraphs $[G, g],[H$, $h] \in \mathcal{M}$ are isomorphic if the following both hold:

$$
\begin{aligned}
& (\forall\langle g, x, y\rangle \in G)(\exists\langle h, a, b\rangle \in H)\left(\left[G_{x}, x\right] \cong\left[H_{a}, a\right] \wedge\left[G_{y}, y\right] \cong\left[H_{b}, b\right]\right), \\
& (\forall\langle h, a, b\rangle \in H)(\exists\langle g, x, y\rangle \in G)\left(\left[G_{x}, x\right] \cong\left[H_{a}, a\right] \wedge\left[G_{y}, y\right] \cong\left[H_{b}, b\right]\right) .
\end{aligned}
$$

Proof Define $x \sim y \Leftrightarrow_{d f}(x=g \wedge y=h) \vee\left[G_{x}, x\right] \cong\left[H_{y}, y\right]$.

One can readily check from the hypothesis that $\sim$ is a bisimulation between $[G, g]$ and $[H, h]$. Since $[G, g],[H, h]$ are accessible and $g \sim h$, by Lemma 4.18 $[G, g] \equiv[H, h]$, but they are extensional so $[G, g] \cong[H, h]$.

Definition 4.28 For any $[H, h] \in \mathcal{M}$ and $y \in H^{-1} x$, write $H(x, y)$ for the unique $z$ such that $H(x, y, z)$.

Definition 4.29 Let $[G, g],[H, h] \in \mathcal{M}$. Say that $[G, g] \bar{\subset}[H, h]$ if there is a relation $\triangleleft \subset \operatorname{Dom}[G, g] \times \operatorname{Dom}[H, h]$ in the ZF model $V$, such that $g \triangleleft h$ and $x \triangleleft y \Rightarrow\left(\forall a \in G^{-1} x\right)\left(\exists b \in H^{-1} y\right)\left(\left[G_{a}, a\right] \cong\left[H_{b}, b\right] \wedge G(x, a) \triangleleft H(y, b)\right)$. 
Lemma 4.30 The relation $\overline{\mathcal{C}}$ respects isomorphisms of multigraphs; that is,

$$
\begin{aligned}
& {[Q, q] \cong[G, g] \wedge[G, g] \bar{\subset}[H, h] \Rightarrow[Q, q] \bar{\subset}[H, h],} \\
& {[Q, q] \bar{\subset}[G, g] \wedge[H, h] \cong[G, g] \Rightarrow[Q, q] \bar{\subset}[H, h] .}
\end{aligned}
$$

Proof If $[Q, q] \cong[G, g]$ by the isomorphism $\varphi$ and $[G, g] \bar{\subset}[H, g]$ as witnessed by the relation $\triangleleft$, then the relation $\varphi(x) \triangleleft y$ witnesses $[Q, q] \bar{\subset}[H, h]$.

The second claim is proved similarly.

The following lemma shows that $\bar{C}$ is precisely the internal inclusion relation of $\mathcal{M}$ as defined in Definition 3.8 and satisfies Axiom 3.1.1.

Lemma 4.31 Let $\varphi(x, y)$ be a formula in the language of multisets with two free variables and possibly parameters, such that

$$
\mathcal{M} \models(\forall x, y)(\varphi(x, y)) \Leftrightarrow(\forall a \bar{\epsilon} x)\left(a \bar{\epsilon} y \wedge \varphi\left(\frac{x}{a}, \frac{y}{a}\right)\right) .
$$

Then $(\forall[Q, q],[H, h] \in \mathcal{M})((\mathcal{M} \models \varphi([Q, q],[H, h])) \Rightarrow[Q, q] \bar{\subset}[H, h])$.

Furthermore, $[G, g] \bar{\subset}[H, h]$ if and only if

$$
(\forall[Q, q] \bar{\epsilon}[G, g])\left([Q, q] \bar{\epsilon}[H, h] \wedge \frac{[G, g]}{[Q, q]} \bar{\subset} \frac{[H, h]}{[Q, q]}\right) .
$$

Finally, $\overline{\mathrm{C}}$ is reflexive, transitive, and

$$
[G, g] \bar{\subset}[H, h] \wedge[H, h] \bar{\subset}[G, g] \Rightarrow[G, g] \cong[H, h],
$$

that is, $\overline{\mathrm{C}}$ is antisymmetric if we interpret $\cong$ as the identity relation.

For clarity we divide the proof into several parts.

Lemma 4.32 Let $\varphi(x, y)$ be a formula in the language of multisets with two free variables and possibly parameters, such that

$$
\mathcal{M} \models(\forall x, y)(\varphi(x, y)) \Leftrightarrow(\forall a \bar{\epsilon} x)\left(a \bar{\epsilon} y \wedge \varphi\left(\frac{x}{a}, \frac{y}{a}\right)\right) .
$$

Then $(\forall[Q, q],[H, h] \in \mathcal{M})((\mathcal{M} \models \varphi([Q, q],[H, h])) \Rightarrow[Q, q] \bar{\subset}[H, h])$.

Proof $\quad$ Suppose $\mathcal{M} \models \varphi([Q, q],[H, h])$. Define a set relation $\triangleleft$ by

$$
x \triangleleft y \Leftrightarrow d f \mathcal{M} \models \varphi\left(\left[Q_{x}, x\right],\left[H_{y}, y\right]\right) .
$$

Then $\triangleleft$ satisfies the condition in Definition 4.29, so $[Q, q] \bar{\subset}[H, h]$.

Definition 4.33 Given any relation $\triangleleft \subset \operatorname{Dom}[G, g] \times \operatorname{Dom}[H, h]$, define $\triangleleft^{+}$by

$$
\begin{aligned}
x \triangleleft^{+} y \Leftrightarrow_{d f}(\forall a & \left.\in G^{-1} x\right)\left(\exists b \in H^{-1} y\right) \\
& \times\left(\left[G_{a}, a\right] \cong\left[H_{b}, b\right] \wedge G(x, a) \triangleleft H(y, b)\right) .
\end{aligned}
$$

Remark 4.34 The operation taking $\triangleleft$ to $\triangleleft^{+}$depends on $[G, g]$ and $[H, h]$, but we omit the associated multigraphs where there is no danger of confusion. Then $\triangleleft_{1} \subset \triangleleft_{2} \Rightarrow \triangleleft_{1}^{+} \subset \triangleleft_{2}^{+}$given the same associated multigraphs.

Definition 4.35 Define the greatest subset relation by

$$
x \prec y \Leftrightarrow d f(\exists \triangleleft \subset \operatorname{Dom}[G, g] \times \operatorname{Dom}[H, h])\left(\triangleleft \subset \triangleleft^{+} \wedge x \triangleleft y\right) .
$$

Remark 4.36 Clearly $[G, g] \bar{\subset}[H, h]$ if and only if $g \prec h$. 
Lemma 4.37 For the greatest subset relation $\prec$ between $[G, g]$ and $[H, h]$,

$$
x \prec y \Leftrightarrow\left(\forall a \in G^{-1} x\right)\left(\exists b \in H^{-1} y\right)\left(\left[G_{a}, a\right] \cong\left[H_{b}, b\right] \wedge G(x, a) \prec H(y, b)\right) .
$$

Proof If $\triangleleft \subset \triangleleft^{+}$and $x \triangleleft y$, then by definition of $\triangleleft^{+}$we have $G(x, a) \prec H(y, b)$ trivially for any $a \in G^{-1} x$ and $b \in H^{-1} y$, so $x \prec^{+} y$. This shows that $\prec \subset \prec^{+}$, so $\prec^{+} \subset \prec^{++}$and thus $\prec=\prec^{+}$by maximality.

Remark 4.38 If $x \in \operatorname{Dom}[G, g], y \in \operatorname{Dom}[H, h]$ and $\triangleleft \subset \operatorname{Dom}\left[G_{x}, x\right] \times$ $\operatorname{Dom}\left[H_{y}, y\right]$, clearly $\triangleleft^{+}$is the same relation whether defined relative to $[G, g]$ and $[H, h]$ or $\left[G_{x}, x\right]$ and $\left[H_{y}, y\right]$. This means the restriction of $\prec$ to $\operatorname{Dom}\left[G_{x}, x\right] \times$ $\operatorname{Dom}\left[H_{y}, y\right]$ is the greatest subset relation between $\left[G_{x}, x\right]$ and $\left[H_{y}, y\right]$; hence

$$
\left[G_{x}, x\right] \bar{\subset}\left[H_{y}, y\right] \Leftrightarrow x \prec y .
$$

Lemma 4.39 For any multigraphs $[G, g]$ and $[H, h]$,

$$
[G, g] \bar{\subset}[H, h] \Leftrightarrow(\forall[Q, q] \bar{\epsilon}[G, g])\left([Q, q] \bar{\epsilon}[H, h] \wedge \frac{[G, g]}{[Q, q]} \bar{\subset} \frac{[H, h]}{[Q, q]}\right) .
$$

Proof Suppose the right-hand side holds. Extend the greatest subset relation $\prec$ between $[G, g]$ and $[H, h]$ to the following:

$$
x \preceq y \Leftrightarrow_{d f} x \prec y \vee(x=g \wedge y=h) .
$$

One can check that $\preceq \subset \preceq^{+}$, so $\preceq=\prec$. This means that $g \prec h$, so $[G, g] \bar{\subset}[H, h]$.

Conversely, let $[G, g] \bar{\subset}[H, h]$ and $[Q, q] \bar{\epsilon}[G, g]$; then $g \prec h$ and $[Q, q] \cong$ $\left[G_{x}, x\right]$ for some $x \in G^{-1} g$. There exists $y \in H^{-1} h$ such that

$$
\left[H_{y}, y\right] \cong\left[G_{x}, x\right] \wedge G(g, x) \prec H(h, y) .
$$

But then $\left[H_{y}, y\right] \cong[Q, q]$, so $[Q, q] \bar{\epsilon}[H, h]$ and

$$
\frac{[G, g]}{[Q, q]} \cong\left[G_{G(g, x)}, G(g, x)\right] \bar{\subset}\left[H_{H(h, y)}, H(H, y)\right] \cong \frac{[H, h]}{[Q, q]},
$$

so $\frac{[G, g]}{[Q, q]} \bar{\subset} \frac{[H, h]}{[Q, q]}$ since $\bar{\subset}$ respects isomorphism.

Lemma 4.40 The relation $\overline{\mathrm{C}}$ is reflexive, transitive, and

$$
[G, g] \bar{\subset}[H, h] \wedge[H, h] \bar{\subset}[G, g] \Rightarrow[G, g] \cong[H, h] ;
$$

that is, $\overline{\mathrm{C}}$ is antisymmetric if we interpret $\cong$ as the identity relation.

Proof If $\triangleleft$ is the identity, then trivially $\triangleleft \subset \triangleleft^{+}$, so $\bar{C}$ is reflexive.

Let $\triangleleft_{1}$ witness $[Q, q] \bar{\subset}[G, g]$, and let $\triangleleft_{2}$ witness $[G, g] \bar{\subset}[H, h]$. Define

$$
x \triangleleft y \Leftrightarrow d f(\exists d)\left(x \triangleleft_{1} d \wedge d \triangleleft_{2} y\right) .
$$

It is straightforward to verify that $\triangleleft \subset \triangleleft^{+}$. Furthermore, $q \triangleleft_{1} g \triangleleft_{2} h$, so $q \triangleleft h$ and thus $\triangleleft$ witnesses $[Q, q] \bar{\subset}[H, h]$. This means that $\bar{C}$ is transitive.

Let $\prec_{1}$ be the greatest subset relation between $[G, g]$ and $[H, h]$, and let $\prec_{2}$ be the greatest subset relation between $[H, h]$ and $[G, g]$. Define $\sim \subset \operatorname{Dom}[G, g] \times$ $\operatorname{Dom}[H, h]$ by

$$
x \sim y \Leftrightarrow d f x \prec_{1} y \wedge y \prec_{2} x .
$$

If $x \sim y$ and $G(x, a, b)$, there exists $c \in H^{-1} y$ such that $\left[G_{a}, a\right] \cong\left[H_{c}, c\right]$ and $b \prec_{1} H(y, c)$. Then $a \sim c$ and $b \sim H(y, c)$. 
Similarly if $x \sim y$ and $H(y, c, d)$, then there are $a \sim c, b \sim d$ such that $G(x, a, b)$. Hence $\sim$ is a bisimulation and $[G, g] \cong[H, h]$.

Thus all claims in Lemma 4.31 have been proved.

4.5 The axiom of union For ease of reference we give the statement of the axiom first, whereas the proof will be divided into several lemmas.

Lemma 4.41 (Axiom of union) $\quad$ Let $[H, h] \in \mathcal{M}$. There exists $[G, g] \in \mathcal{M}$ such that the following both hold:

$$
\begin{gathered}
(\forall[Q, q] \bar{\epsilon}[H, h])[Q, q] \bar{\subset}[G, g], \\
(\forall[P, p] \in \mathcal{M})((\forall[Q, q] \bar{\epsilon}[H, h])[Q, q] \bar{\subset}[P, p] \Rightarrow[G, g] \bar{\subset}[P, p]) .
\end{gathered}
$$

Remark 4.42 We will define a recursive relation between vertices of $[G, g]$ and sets of vertices of $[H, h]$. Intuitively the vertex $a$ is related to the set $X$ if the subgraph $\left[G_{a}, a\right]$ is the $\bar{C}$-least upper bound of $\left\{\left[H_{x}, x\right]: x \in X\right\}$. Hence $[G, g]$ is the union of $[H, h]$ if and only if $g$ is related to $H^{-1} h$.

Let $[G, g]$ and $[H, h]$ be fixed, and define (see Lemma 3.47) the following.

Definition 4.43 For any relation $\triangleleft \subset \operatorname{Dom}[G, g] \times \mathcal{P}(\operatorname{Dom}[H, h])$ define

$$
\begin{aligned}
a \triangleleft^{+} X \Leftrightarrow d f( & \forall[Q, q] \in \mathcal{M})\left([Q, q] \bar{\epsilon}\left[G_{a}, a\right] \Leftrightarrow(\exists x \in X)[Q, q] \bar{\epsilon}\left[H_{x}, x\right]\right) \\
& \wedge\left(\forall b \in G^{-1} a\right) G(a, b) \triangleleft\{z:(\exists x \in X)(\exists y)(H(x, y, z) \\
& \left.\left.\wedge\left[G_{b}, b\right] \cong\left[H_{y}, y\right]\right)\right\} .
\end{aligned}
$$

The recursive relation we mentioned is the following.

Definition 4.44 For any $a \in \operatorname{Dom}[G, g]$ and $X \subset \operatorname{Dom}[H, h]$ let

$$
a \prec X \Leftrightarrow d f(\exists \triangleleft \subset \operatorname{Dom}[G, g] \times \mathcal{P}(\operatorname{Dom}[H, h]))\left(\triangleleft \subset \triangleleft^{+} \wedge a \triangleleft X\right) .
$$

Remark 4.45 It is straightforward to see that $\triangleleft_{1} \subset \triangleleft_{2} \Rightarrow \triangleleft_{1}^{+} \subset \triangleleft_{2}^{+}$. As before, by the Knaster-Tarski theorem we have $\prec=\prec^{+}$.

Lemma 4.46 For each $X \subset \operatorname{Dom}[H, h]$ there is no more than one $a \prec X$.

Proof Define a relation $\sim$ on $\operatorname{Dom}[G, g]$ by

$$
a \sim b \Leftrightarrow_{d f}(\exists X \subset \operatorname{Dom}[H, h])(a \prec X \wedge b \prec X) .
$$

If $a \sim b$, then $(\forall[Q, q] \in \mathcal{M})\left([Q, q] \bar{\epsilon}\left[G_{a}, a\right] \Leftrightarrow[Q, q] \bar{\epsilon}\left[G_{b}, b\right]\right)$.

But $[G, g]$ is extensional, so $G^{-1} a=G^{-1} b$. Now let $c \in G^{-1} a$; then

$$
\begin{aligned}
& G(a, c) \prec\left\{z:(\exists x \in X)(\exists y)\left(H(x, y, z) \wedge\left[G_{c}, c\right] \cong\left[H_{y}, y\right]\right)\right\}, \\
& G(b, c) \prec\left\{z:(\exists x \in X)(\exists y)\left(H(x, y, z) \wedge\left[G_{c}, c\right] \cong\left[H_{y}, y\right]\right)\right\},
\end{aligned}
$$

so $G(a, c) \sim G(b, c)$. Hence $\sim$ is a bisimulation on $[G, g]$, so it is the identity.

Lemma 4.47 If $g \prec H^{-1} h$, then $[G, g]$ is the supposed multiset union of $[H, h]$ (i.e., they satisfy the condition in Lemma 4.41). 
Proof First let $[Q, q] \bar{\epsilon}[H, h]$; then $[Q, q] \cong\left[H_{d}, d\right]$ for some $d \in H^{-1} h$.

Define $\triangleleft \subset \operatorname{Dom}[H, h] \times \operatorname{Dom}[G, g]$ to witness $\left[H_{d}, d\right] \bar{C}[G, g]$ by

$$
x \triangleleft y \Leftrightarrow_{d f}(\exists X \subset \operatorname{Dom}[H, h])(x \in X \wedge y \prec X) .
$$

If $x \triangleleft y$, then by the defining property of $\prec$,

$$
\left(\forall[Q, q] \bar{\epsilon}\left[H_{x}, x\right]\right)[Q, q] \bar{\epsilon}\left[G_{y}, y\right],
$$

so for any $a \in H^{-1} x$ there exists $b \in G^{-1} y$ such that $\left[H_{a}, a\right] \cong\left[G_{b}, b\right]$.

Let $X$ witness $x \triangleleft y$; then $H(x, a) \triangleleft G(y, b)$ since the following both hold:

$$
\begin{aligned}
& H(x, a) \in\left\{z:(\exists x \in X)(\exists y)\left(H(x, y, z) \wedge\left[G_{b}, b\right] \cong\left[H_{y}, y\right]\right)\right\}, \\
& G(y, b) \prec\left\{z:(\exists x \in X)(\exists y)\left(H(x, y, z) \wedge\left[G_{b}, b\right] \cong\left[H_{y}, y\right]\right)\right\} .
\end{aligned}
$$

Trivially $d \triangleleft g$, so $\triangleleft$ witnesses $\left[H_{d}, d\right] \bar{\subset}[G, g]$ and thus $[Q, q] \bar{\subset}[G, g]$.

Conversely, suppose $[P, p]$ is such that $[Q, q] \bar{\subset}[P, p]$ for any $[Q, q] \bar{\epsilon}[H, h]$.

Define $\triangleleft \subset \operatorname{Dom}[G, g] \times \operatorname{Dom}[P, p]$ to witness $[G, g] \bar{\subset}[P, p]$ by

$$
a \triangleleft b \Leftrightarrow d f(\exists X \subset \operatorname{Dom}[H, h])\left(a \prec X \wedge(\forall x \in X)\left[H_{x}, x\right] \bar{\subset}\left[P_{b}, b\right]\right) .
$$

First note that $g \triangleleft p$ as witnessed by $H^{-1} h$.

Let $c \in G^{-1} a$, and let $X$ witness $a \triangleleft b$. Since $a \prec X,\left[G_{c}, c\right] \bar{\epsilon}\left[H_{x}, x\right]$ for some $x \in X$. But $\left[H_{x}, x\right] \bar{\subset}\left[P_{b}, b\right]$, so $\left[G_{c}, c\right] \cong\left[P_{v}, v\right]$ for some $v \in P^{-1} b$.

Also $G(a, c) \prec Y$ by the recursive property of $\prec$ where

$$
Y:=\left\{y:(\exists d \in X)(\exists e)\left(H(d, e, y) \wedge\left[G_{c}, c\right] \cong\left[H_{e}, e\right]\right)\right\} .
$$

Suppose that $d, e$ witness $y \in Y$ as above; then $\left[H_{e}, e\right] \cong\left[P_{v}, v\right]$. But $d \in X$, so $\left[H_{d}, d\right] \bar{\subset}\left[P_{b}, b\right]$; hence $\left[H_{y}, y\right] \bar{\subset}\left[P_{P(b, v)}, P(b, v)\right]$ by the property of $\overline{\mathcal{C}}$. Thus

$$
(\forall y \in Y)\left[H_{w}, w\right] \bar{\subset}\left[P_{P(b, v)}, P(b, v)\right] .
$$

Therefore $G(a, c) \triangleleft P(b, v)$, and so $\triangleleft$ witnesses $[G, g] \bar{\subset}[P, p]$, as required.

Thus it suffices to construct a multigraph $[G, g]$ such that $g \prec H^{-1} h$, where $\prec$ is defined as in Definition 4.44. Intuitively we add a new vertex $v(X)$ for each $X \subset \operatorname{Dom}[H, h]$ and build a new graph $D$ recursively so that the extensional quotient of $\left[D_{v(X)}, v(X)\right]$ is the $\bar{C}$-least upper bound of $\left\{\left[H_{x}, x\right]: x \in X\right\}$. Then we let $d:=v\left(H^{-1} h\right)$, and the extensional quotient of $[D, d]$ will be the required $[G, g]$.

Definition 4.48 Let $v$ be a fixed bijection from $\mathcal{P}(\operatorname{Dom}[H, h])$ to some set $A$ disjoint from $\operatorname{Dom}[H, h]$, and let $d:=v\left(H^{-1} h\right)$.

Remark 4.49 Since our underlying set-theoretic model is a model of ZF, it is trivial to obtain a uniform definition for $v$ given any $[H, h]$.

The range $A$ of $v$ will be the set of new vertices corresponding to subsets of $\operatorname{Dom}[H, h]$, and we will build a graph $C$ in which each vertex in $A$ represents the union of its preimage under $v$.

Definition 4.50 Let $C$ be the smallest set such that

$$
\left(\forall b \in \bigcup\left\{H^{-1} a: a \in H^{-1} h\right\}\right)\left\langle d, b, v\left(\left\{c:\left(\exists a \in H^{-1} h\right) H(a, b, c)\right\}\right)\right\rangle \in C,
$$

and proceed similarly for any $X \subset \operatorname{Dom}[H, h]$ such that $v(X) \in \operatorname{Dom} C$,

$$
\left(\forall b \in \bigcup\left\{H^{-1} a: a \in X\right\}\right)\langle v(X), b, v(\{c:(\exists a \in X) H(a, b, c)\})\rangle \in C .
$$

Note that the unions in this definition are set-theoretic. 
Informally $C$ is built by following the recursive property of a multiset union on multiplicities (see Lemma 3.47) from the top vertex $d$ down. In the definition of $C$ above, the vertex $b$ represents members of the union, their members, members of their members, and so on; we simply copy the corresponding subgraphs of $[H, h]$ over to form the final hypergraph, that is, so that $\left[C_{b}, b\right]=\left[H_{b}, b\right]$.

Definition 4.51 Let

$$
D:=C \cup \bigcup\left\{H_{b}:(\exists x, y \in A) C(x, b, y)\right\} .
$$

Let $[G, g]$ be the extensional quotient of $[D, d]$, and let $\pi$ be the quotient map.

Note that if $C(x, b, y)$, then $(\nexists v, w) C(b, v, w)$. Hence $\left[D_{b}, b\right]=\left[H_{b}, b\right]$.

Lemma 4.52 We have $[G, g] \in \mathcal{M}$.

Proof Note that the subgraph $C_{d}=C$ by minimality of $C$; thus $[C, d]$ is accessible. If $a \in \operatorname{Dom} H_{b}$ where $(\exists x, y \in A)\langle x, b, y\rangle \in C$, then $a$ is in a finite directed path from $d$ through $b$, so $[D, d]$ is accessible.

Now suppose $D(x, a, v)$ and $D(x, b, w)$ where $\left[D_{a}, a\right] \equiv\left[D_{b}, b\right]$.

By construction $\left[D_{a}, a\right]=\left[H_{a}, a\right]$ and $\left[D_{b}, b\right]=\left[H_{b}, b\right]$, but $[H, h]$ is extensional, so $a=b$. By construction of $[D, d]$ there is a unique $v$ such that $D(x, a, v)$, so $v=w$. Hence $[D, d]$ satisfies the conditions of Lemma 4.22 and $[G, g] \in \mathcal{M}$.

Lemma 4.53 We have $g \prec H^{-1} h$ where $\prec$ is as defined in Definition 4.44.

Proof Define $a \triangleleft X \Leftrightarrow_{d f} a=\pi v(X)$. We first show that $\triangleleft \subset \triangleleft^{+}$.

Let $a=\pi v(X)$. If $v \in H^{-1} x$ for some $x \in X$, then

$$
\langle v(X), v, v\{w:(\exists x \in X) H(x, v, w)\}\rangle \in D .
$$

Hence $\pi(v) \in G^{-1} a$. But $\left[D_{v}, v\right]=\left[H_{v}, v\right]$ is extensional, so $\left[G_{\pi(v)}, \pi(v)\right] \cong$ $\left[H_{v}, v\right]$ by the quotient lemma.

Conversely, if $b \in G^{-1} a$, then $b=\pi(v)$ where $v \in H^{-1} x$ for some $x \in X$. But then $\left[D_{v}, v\right]=\left[H_{v}, v\right]$ is extensional, so $\left[G_{b}, b\right] \cong\left[H_{v}, v\right]$.

We have shown that for any $Q \in \mathcal{M}$,

$$
[Q, q] \bar{\epsilon}\left[G_{a}, a\right] \Leftrightarrow(\exists x \in X)[Q, q] \bar{\epsilon}\left[H_{x}, x\right] .
$$

Also with $b=\pi(v)$, by construction $G(a, b)=\pi v(\{w:(\exists x \in X) H(x, v, w)\})$.

But $v$ is the unique $y$ such that $\left[H_{y}, y\right] \cong\left[G_{b}, b\right]$ since $[H, h]$ is extensional, so

$$
\begin{aligned}
\{w & :(\exists x \in X) H(x, v, w)\} \\
& =\left\{w:(\exists x \in X)(\exists y)\left(H(x, y, w) \wedge\left[G_{b}, b\right] \cong\left[H_{y}, y\right]\right)\right\} .
\end{aligned}
$$

Hence $a \triangleleft^{+} X$ by definition. But $g \triangleleft H^{-1} h$, so $g \prec H^{-1} h$, as required.

This concludes the proof of Lemma 4.41.

4.6 Supertransitivity The following lemma shows that $\mathcal{M}$ is in a sense supertransitive; that is, for any set $X \subset \mathcal{M}$ there is a multigraph whose members in the sense of $\mathcal{M}$ are precisely members of $X$ in $V$.

Lemma 4.54 Let $\varphi$ be a function in $V$ such that $\operatorname{dom} \varphi, \operatorname{ran} \varphi \subset \mathcal{M}$ and $(\forall[G, g],[H, h] \in \operatorname{dom} \varphi)[G, g] \cong[H, h] \Rightarrow \varphi[G, g]=\varphi[H, h]$. 
Then there exists $[Q, q] \in \mathcal{M}$ such that the following both hold:

$$
\begin{gathered}
(\forall[H, h] \in \mathcal{M})([H, h] \bar{\epsilon}[Q, q] \Leftrightarrow(\exists[G, g] \in \operatorname{dom} \varphi)[G, g] \cong[H, h]), \\
(\forall[H, h] \in \operatorname{dom} \varphi) \frac{[Q, q]}{[H, h]} \cong \varphi[H, h] .
\end{gathered}
$$

Proof If $[H, h] \in \operatorname{dom} \varphi \cup \operatorname{ran} \varphi$, define $v[H, h] \in \mathcal{M}$ as follows.

- If $a \in \operatorname{Dom}[H, h]$ let $\hat{a}:=\langle a,[H, h]\rangle$ and $\hat{H}:=\{\langle\hat{a}, \hat{b}, \hat{c}\rangle:\langle a, b, c\rangle \in H\}$.

- Let $v[H, h]:=[\hat{H}, \hat{h}]$; then clearly $v[H, h] \cong[H, h]$.

Furthermore, if $[G, g]$ and $[H, h]$ are different multigraphs, then dom $v[G, g]$ and dom $v[H, h]$ are disjoint. Thus we can assume without loss of generality that distinct multigraphs in $\operatorname{dom} \varphi \cup \operatorname{ran} \varphi$ have disjoint domains.

Let $d \notin \operatorname{Dom}[G, g]$ for all $[G, g] \in \operatorname{dom} \varphi \cup \operatorname{ran} \varphi$. Let

$$
D:=\bigcup\{G:[G, g] \in \operatorname{dom} \varphi \cup \operatorname{ran} \varphi\} \cup\{\langle d, g, h\rangle:(\exists G, H)[H, h]=\varphi[G, g]\},
$$

and let $[Q, q]$ be the extensional quotient of $[D, d]$ with quotient map $\pi$.

It is easy to check that $[D, d]$ is accessible and $[Q, q]$ is a multigraph.

If $[H, h] \bar{\epsilon}[Q, q]$, then $[H, h] \cong\left[Q_{\pi}(a), \pi(a)\right]$ for some $[A, a] \in \operatorname{dom} \varphi$.

Since members of $\operatorname{dom} \varphi \cup \operatorname{ran} \varphi$ have disjoint domains, $\left[D_{a}, a\right]=[A, a]$ by construction. But $[A, a]$ is extensional, so $\left[Q_{\pi}(a), \pi(a)\right] \cong[A, a]$, and thus $[H, h] \cong[A, a]$.

Conversely, let $[H, h] \in \operatorname{dom} \varphi$; then $\left[D_{h}, h\right]=[H, h]$, and thus $\left[Q_{\pi}(h), \pi(h)\right] \cong$ $[H, h]$ since $[H, h]$ is extensional. Let $[A, a]:=\varphi([H, h])$; then

$$
[H, h] \bar{\epsilon}[Q, q] \wedge \frac{[Q, q]}{[H, h]}=\left[Q_{\pi}(a), \pi(a)\right] .
$$

But also $\left[D_{a}, a\right]=[A, a]$ and $\left[Q_{\pi}(a), \pi(a)\right] \cong[A, a]$, so $\frac{[Q, q]}{[H, h]} \cong \varphi[H, h]$.

4.7 The axiom of power set We aim to obtain the $\mathcal{M}$-power set of $[H, h]$ by supertransitivity. For any $[H, h] \in \mathcal{M}$ we will define a subset of the class $\mathcal{M}$ which contains an isomorphic copy of every $[Q, q] \bar{\subset}[H, h]$.

Definition 4.55 An edge chain in $[H, h]$ is a sequence of linked edges of $H$, that is, $\left\langle h_{2 i-1}, h_{2 i}, h_{2 i+1}\right\rangle: 1 \leq i \leq n$, where $h_{1}=h$ and $n \geq 1$. We can denote such an edge chain by the vertex sequence $\left\lfloor h_{1} \cdots h_{2 n+1}\right\rfloor$ without ambiguity.

The expansion $\exp [H, h]$ is the set of all edge chains in $[H, h]$.

Lemma 4.56 Two edge chains $\left\lfloor g_{1} \cdots g_{2 n+1}\right\rfloor$ and $\left\lfloor h_{1} \cdots h_{2 n+1}\right\rfloor$ in $[H, h]$ are identical if $\left[H_{g_{2 i}}, g_{2 i}\right] \cong\left[H_{h_{2 i}}, h_{2 i}\right]$ for all $1 \leq i \leq n$.

Proof Since $[H, h]$ is extensional, the hypothesis means that $g_{2 i}=h_{2 i}$ for all $1 \leq i \leq n$. But $g_{1}=h=h_{1}$, so the result follows by induction on $n$ and uniqueness of multiplicity.

Lemma 4.57 If $[Q, q] \bar{\subset}[H, h]$, then for any $\left\lfloor q_{1} \cdots q_{2 n+1}\right\rfloor \in \exp [Q, q]$ there exists $\left\lfloor h_{1} \cdots h_{2 n+1}\right\rfloor \in \exp [H, h]$ such that $\left[Q_{q_{2 i}}, q_{2 i}\right] \cong\left[H_{h_{2 i}}, h_{2 i}\right]$ and $\left[Q_{q_{2 i+1}}, q_{2 i+1}\right] \bar{\subset}\left[H_{h_{2 i+1}}, h_{2 i+1}\right]$ for all $1 \leq i \leq n$.

Proof This follows by induction on $n$ using Lemma 4.31 in the induction step. 
Definition 4.58 Let $A \subset \exp [Q, q]$ and $B \subset \exp [H, h]$. A projection $\varphi: A \rightarrow B$ is an injection which preserves chain length, that is,

$$
\varphi\left\lfloor q_{1} \cdots q_{2 n+1}\right\rfloor=\left\lfloor h_{1} \cdots h_{2 m+1}\right\rfloor \Rightarrow m=n,
$$

and is such that the following conditions hold.

- If $\varphi\left\lfloor q_{1} \cdots q_{2 n-1}\right\rfloor=\left\lfloor h_{1} \cdots h_{2 n-1}\right\rfloor$ and $\left\langle q_{2 n-1}, q_{2 n}, q_{2 n+1}\right\rangle \in Q$, then $\varphi\left\lfloor q_{1} \cdots q_{2 n+1}\right\rfloor=\left\lfloor h_{1} \cdots h_{2 n-1}, h_{2 n}, h_{n+1}\right\rfloor$ for some $h_{2 n}, h_{2 n+1} \in$ $\operatorname{Dom}[H, h]$.

- If $\varphi\left\lfloor q_{1} \cdots q_{2 n+1}\right\rfloor=\left\lfloor h_{1} \cdots h_{2 n+1}\right\rfloor$, then $\left[Q_{q_{2 n}}, q_{2 n}\right] \cong\left[H_{h_{2 n}}, h_{2 n}\right]$.

The next result follows trivially from the definition.

Corollary 4.59 Let $A \subset \exp [Q, q], B \subset \exp [H, h]$, and $C \subset \exp [G, g]$.

If $\varphi: A \rightarrow B$ and $\psi: B \rightarrow C$ are projections, then the composite $\psi \varphi: A \rightarrow C$ is a projection.

Lemma 4.60 Let $A \subset \exp [Q, q], B \subset \exp [H, h]$, and let $\varphi: A \rightarrow B$ be a projection.

$$
\begin{aligned}
& \text { If } \varphi\left\lfloor q_{1} \cdots q_{2 n+1}\right\rfloor=\left\lfloor h_{1} \cdots h_{2 n+1}\right\rfloor \text {, then for any } 1 \leq i \leq n, \\
& \qquad \varphi\left\lfloor q_{1} \cdots q_{2 i+1}\right\rfloor=\left\lfloor h_{1} \cdots h_{2 i+1}\right\rfloor \wedge\left[Q_{q_{2 i}}, q_{2 i}\right] \cong\left[H_{h_{2 i}}, h_{2 i}\right] .
\end{aligned}
$$

Proof We prove the claim for $i=n-1$ from the case $i=n$ (which is true by definition); then an induction on $i$ with the same argument in the inductive step will complete the proof. Let $\varphi\left\lfloor q_{1} \cdots q_{2 n-1}\right\rfloor=\left\lfloor g_{1} \cdots g_{2 n-1}\right\rfloor$; then by the first condition of Definition 4.58, $\varphi\left\lfloor q_{1} \cdots q_{2 n+1}\right\rfloor=\left\lfloor g_{1} \cdots g_{2 n+1}\right\rfloor$ for some $g_{2 n}, g_{2 n+1}$. Hence $g_{m}=h_{m}$ for all $1 \leq m \leq 2 n+1$. By the second condition of Definition 4.58 we then have $\left[Q_{q_{2 n}}, q_{2 n}\right] \cong\left[H_{h_{2 n-2}}, h_{2 n-2}\right]$.

Lemma 4.61 Let $A \subset \exp [Q, q], B \subset \exp [H, h]$, and let $\varphi: A \rightarrow B$ be $a$ projection.

Then the inverse $\varphi^{-1}: \operatorname{ran} \varphi \rightarrow A$ is also a projection.

Proof Clearly $\varphi^{-1}$ preserves chain length since $\varphi$ does, and the second condition in Definition 4.58 is also trivially satisfied.

Suppose $\varphi\left\lfloor q_{1} \cdots q_{2 n-1}\right\rfloor=\left\lfloor h_{1} \cdots h_{2 n-1}\right\rfloor$ and $\left\langle h_{2 n-1}, h_{2 n}, h_{2 n+1}\right\rangle \in H$. Let $\left\lfloor g_{1} \cdots g_{2 n+1}\right\rfloor=\varphi^{-1}\left\lfloor h_{1} \cdots h_{2 n+1}\right\rfloor$; then by Lemma 4.60,

$$
\left\lfloor g_{1} \cdots g_{2 n-1}\right\rfloor=\varphi^{-1}\left\lfloor h_{1} \cdots h_{2 n-1}\right\rfloor=\left\lfloor q_{1} \cdots q_{2 n-1}\right\rfloor .
$$

Hence the first condition in Definition 4.58 also holds.

Lemma 4.62 We have $[Q, q] \bar{\subset}[H, h]$ if and only if there is a projection $\varphi: \exp [Q, q] \rightarrow \exp [H, h]$.

Proof For the first direction suppose $[Q, q] \overline{\mathrm{C}}[H, h]$.

Define $\varphi\left\lfloor q_{1} \cdots q_{2 n+1}\right\rfloor:=\left\lfloor h_{1} \cdots h_{2 n+1}\right\rfloor$ if $\left[Q_{q_{2 i}}, q_{2 i}\right] \bar{\subset}\left[H_{h_{2 i+1}}, h_{2 i+1}\right]$ and $\left[Q q_{2 i}, q_{2 i}\right] \cong\left[H_{h_{2 i}}, h_{2 i}\right]$ for all $1 \leq i \leq n$. By Lemmas 4.56 and $4.57, \varphi$ is a welldefined function on all of $\exp [Q, q]$; and the second condition in Definition 4.58 holds trivially.

If $\varphi\left\lfloor q_{1} \cdots q_{2 n+1}\right\rfloor=\varphi\left\lfloor b_{1} \cdots b_{2 m+1}\right\rfloor$, then $m=n$ since $\varphi$ preserves chain length by definition. Furthermore, $\left[Q_{q_{2 i}}, q_{2 i}\right] \cong\left[Q_{b_{2 i}}, b_{2 i}\right]$ for all $1 \leq i \leq n$, so by Lemma 4.56, $\left\lfloor q_{1} \cdots q_{2 n+1}\right\rfloor=\left\lfloor b_{1} \cdots b_{2 m+1}\right\rfloor$, and thus $\varphi$ is injective. 


$$
\text { If } \begin{aligned}
\varphi\left\lfloor q_{1} \cdots q_{2 n-1}\right\rfloor= & \left\lfloor h_{1} \cdots h_{2 n-1}\right\rfloor \text { and }\left\langle q_{2 n-1}, q_{2 n}, q_{2 n+1}\right\rangle \in Q \text {, let } \\
& \left\lfloor b_{1} \cdots b_{2 n+1}\right\rfloor:=\varphi\left\lfloor q_{1} \cdots q_{2 n+1}\right\rfloor .
\end{aligned}
$$

Then $\left\lfloor b_{1} \cdots b_{2 n-1}\right\rfloor=\varphi\left\lfloor q_{1} \cdots q_{2 n-1}\right\rfloor$ by checking the definition of $\varphi$, so $\left\lfloor b_{1} \cdots b_{2 n-1}\right\rfloor=\left\lfloor h_{1} \cdots h_{2 n-1}\right\rfloor$. Thus the first condition of Definition 4.58 holds, and $\varphi$ is a projection.

Conversely, suppose that there exists a projection $\varphi: \exp [Q, q] \rightarrow \exp [H, h]$.

Define $x \triangleleft y$ if there exists $\varphi\left\lfloor q_{1} \cdots q_{2 n+1}\right\rfloor=\left\lfloor h_{1} \cdots h_{2 n+1}\right\rfloor$ such that $x=q_{2 n+1}$ and $y=h_{2 n+1}$, or $x=q$ and $y=h$.

Suppose $n>1$ and $\varphi\left\lfloor q_{1} \cdots q_{2 n-1}\right\rfloor=\left\lfloor h_{1} \cdots h_{2 n-1}\right\rfloor$ and $\left\langle q_{2 n-1}, q_{2 n}\right.$, $\left.q_{2 n+1}\right\rangle \in Q$. By the first condition of Definition 4.58 we can extend $\left\lfloor h_{1} \cdots h_{2 n-1}\right\rfloor$ to $\left\lfloor h_{1} \cdots h_{2 n+1}\right\rfloor=\varphi\left\lfloor q_{1} \cdots q_{2 n+1}\right\rfloor$.

If $n=1$, just take $\left\lfloor h_{1} \cdots h_{2 n+1}\right\rfloor:=\varphi\left\lfloor q_{1} \cdots q_{2 n+1}\right\rfloor$ since there is nothing to extend.

By the second condition of Definition 4.58, $\left[H_{h_{2 n}}, h_{2 n}\right] \cong\left[Q_{q_{2 n}}, q_{2 n}\right]$, while $q_{2 n+1} \triangleleft h_{2 n+1}$ by definition and $\left\langle h_{2 n-1}, h_{2 n}, h_{2 n+1}\right\rangle \in H$.

Hence $\triangleleft$ witnesses $[Q, q] \bar{\subset}[H, h]$ as in Definition 4.29.

Definition 4.63 Say that $B \subset \exp [H, h]$ is a subimage of $[H, h]$ if it is closed under initial subchains, that is, if $\left\lfloor h_{1} \cdots h_{2 n+1}\right\rfloor \in B$ where $n>1$, then $\left\lfloor h_{1} \cdots h_{2 n-1}\right\rfloor \in B$.

We will establish a correspondence between the subimages of $[H, h]$ and the submultisets of $[H, h]$ in our interpretation of multiset theory on $\mathcal{M}$. To this end we first define a unique pointed hypergraph for each subimage.

Definition 4.64 Write $\left\lceil x_{1} \cdots x_{n}\right\rceil$ for an arbitrary finite sequence, not necessarily in any expansion.

For each multigraph $[H, h]$ choose a fixed injection $v$ where dom $v$ is the set of all finite vertex sequences of $[H, h]$, and $\operatorname{ran} v$ is disjoint from $\operatorname{Dom}[H, h]$. Since $[H, h]$ lives in a model of $\mathrm{ZF}$, we can find a uniform definition for $v$ in terms of $[H, h]$ by utilizing foundation.

Definition 4.65 If $\left\lfloor h_{1} \cdots h_{2 n+1}\right\rfloor \in \exp [H, h]$, define

$$
\begin{aligned}
H\left\lceil h_{1} \cdots h_{2 n}\right\rceil:= & \left\{\left\langle v\left\lceil h_{1} \cdots h_{2 n-1}, a\right\rceil, v\left\lceil h_{1} \cdots h_{2 n-1}, b\right\rceil, v\left\lceil h_{1} \cdots h_{2 n-1}, c\right\rceil\right\rangle:\right. \\
& \left.\langle a, b, c\rangle \in H_{h_{2 n}}\right\} .
\end{aligned}
$$

The point of the definition above is that $\left[H\left\lceil h_{1} \cdots h_{2 n}\right\rceil, v\left\lceil h_{1} \cdots h_{2 n}\right\rceil\right]$ is an isomorphic copy of $\left[H_{h_{2 n}}, h_{2 n}\right]$, and these new multigraphs are all disjoint for different $\left\lfloor h_{1} \cdots h_{2 n+1}\right\rfloor \in \exp [H, h]$.

Definition 4.66 For any subimage $B$ of $[H, h]$, define

$$
\begin{aligned}
\bar{B}:= & \left\{\left\langle\nu\left\lceil h_{1} \cdots h_{2 n-1}\right\rceil, v\left\lceil h_{1} \cdots h_{2 n}\right\rceil, v\left\lceil h_{1} \cdots h_{2 n+1}\right\rceil\right\rangle:\left\lfloor h_{1} \cdots h_{2 n+1}\right\rfloor \in B\right\} \\
& \cup \bigcup\left\{H\left\lceil h_{1} \cdots h_{2 n}\right\rceil:\left\lfloor h_{1} \cdots h_{2 n+1}\right\rfloor \in B\right\} .
\end{aligned}
$$

Intuitively $\bar{B}$ is the graph created by first making a tree where the edge chains in $B$ are glued together at their common initial subchains, then attaching to each even-indexed vertex $v\left\lceil h_{1} \cdots h_{2 n}\right\rceil$ the subgraph $H\left\lceil h_{1} \cdots h_{2 n}\right\rceil$.

Lemma 4.67 The pointed hypergraph $[\bar{B}, v\lceil h\rceil]$ is accessible. 
Proof Let $x$ be a vertex of $[\bar{B}, v\lceil h\rceil]$; then it falls into one of two cases.

If $x$ is in the edge $\left\langle v\left\lceil h_{1} \cdots h_{2 n-1}\right\rceil, v\left\lceil h_{1} \cdots h_{2 n}\right\rceil, v\left\lceil h_{1} \cdots h_{2 n+1}\right\rceil\right\rangle$ where $\left\lfloor h_{1} \cdots h_{2 n+1}\right\rfloor \in B$, the following finite directed path goes from $v\lceil h\rceil$ to $x$ :

$$
\left\langle v\left\lceil h_{1} \cdots h_{2 i-1}\right\rceil, v\left\lceil h_{1} \cdots h_{2 i}\right\rceil, v\left\lceil h_{1} \cdots h_{2 i+1}\right\rceil\right\rangle: i=1 \cdots n .
$$

If $x \in \operatorname{Dom} H\left\lceil h_{1} \cdots h_{2 n}\right\rceil$, there is a finite directed path from $v\left\lceil h_{1} \cdots h_{2 n}\right\rceil$ to $x$ since $H\left\lceil h_{1} \cdots h_{2 n}\right\rceil \cong H_{h_{2 n}}$ (see Definition 4.65). Hence there is a path from $v\lceil h\rceil$ to $x$.

Lemma 4.68 If $\left\lfloor h_{1} \cdots h_{2 n+1}\right\rfloor \in B$, then $\left[\bar{B}_{v\left\lceil h_{1} \cdots h_{2 n}\right\rceil}, v\left\lceil h_{1} \cdots h_{2 n}\right\rceil\right] \cong\left[H_{h_{2 n}}\right.$, $\left.h_{2 n}\right]$.

Proof On the other hand, Dom $H\left\lceil h_{1} \cdots h_{2 n}\right\rceil$ is disjoint from Dom $H\left\lceil g_{1} \cdots g_{2 n}\right\rceil$ for any other sequence $\left\lceil g_{1} \cdots g_{2 n}\right\rceil$, and except for the top vertex $v\left\lceil h_{1} \cdots h_{2 n}\right\rceil$ it is also disjoint from the tree

$$
\left\{\left\langle v\left\lceil h_{1} \cdots h_{2 n-1}\right\rceil, v\left\lceil h_{1} \cdots h_{2 n}\right\rceil, v\left\lceil h_{1} \cdots h_{2 n+1}\right\rceil\right\rangle:\left\lfloor h_{1} \cdots h_{2 n+1}\right\rfloor \in B\right\} .
$$

Now $H\left\lceil h_{1} \cdots h_{2 n}\right\rceil$ is accessible from $v\left\lceil h_{1} \cdots h_{2 n}\right\rceil$ (see Lemma 4.67), so

$$
\left[\bar{B}_{v\left\lceil h_{1} \cdots h_{2 n}\right\rceil}, v\left\lceil h_{1} \cdots h_{2 n}\right\rceil\right]=\left[H\left\lceil h_{1} \cdots h_{2 n}\right\rceil, v\left\lceil h_{1} \cdots h_{2 n}\right\rceil\right],
$$

but $\left[H\left\lceil h_{1} \cdots h_{2 n}\right\rceil, v\left\lceil h_{1} \cdots h_{2 n}\right\rceil\right] \cong\left[H_{h_{2 n}}, h_{2 n}\right]$, so the result follows.

Lemma 4.69 The pointed hypergraph $[\bar{B}, v\lceil h\rceil]$ satisfies the hypothesis of Lemma 4.22; hence its extensional quotient is a multigraph.

Proof We have shown that $[\bar{B}, v\lceil h\rceil]$ is accessible.

Suppose $\bar{B}(x, a, v)$ and $\bar{B}(x, b, w)$, where $\left[\bar{B}_{a}, a\right] \equiv\left[\bar{B}_{b}, b\right]$. We prove $v=w$ by considering the two cases with $x$ again.

If $x=v\left\lceil h_{1} \cdots h_{2 n+1}\right\rceil$ where $\left\lfloor h_{1} \cdots h_{2 n+1}\right\rfloor \in B$, there are $a_{0}, b_{0} \in \operatorname{Dom}[H, h]$ such that $a=v\left\lceil h_{1} \cdots h_{2 n+1}, a_{0}\right\rceil$ and $b=v\left\lceil h_{1} \cdots h_{n}, b_{0}\right\rceil$.

By Lemma $4.68\left[\bar{B}_{a}, a\right] \cong\left[H_{a_{0}}, a_{0}\right]$ and $\left[\bar{B}_{b}, b\right] \cong\left[H_{b_{0}}, b_{0}\right]$, so $\left[H_{a_{0}}, a_{0}\right] \equiv$ $\left[H_{b_{0}}, b_{0}\right]$, and thus $a_{0}=b_{0}$ since $[H, h]$ is extensional. By construction of $\bar{B}$ we have

$v=v\left\lceil h_{1} \cdots h_{2 n+1}, a_{0}, H\left(h_{2 n+1}, a_{0}\right)\right\rceil=v\left\lceil h_{1} \cdots h_{2 n+1}, b_{0}, H\left(h_{2 n+1}, b_{0}\right)\right\rceil=w$.

If $x \in \operatorname{Dom} H\left\lceil h_{1} \cdots h_{2 n}\right\rceil$ where $\left\lfloor h_{1} \cdots h_{2 n+1}\right\rfloor \in B$ (this case includes $\left.x=v\left\lceil h_{1} \cdots h_{2 n}\right\rceil\right)$, then $\left[\bar{B}_{x}, x\right] \in \mathcal{M}$ by Lemma 4.68.

But $\left[\bar{B}_{a}, a\right] \equiv\left[\bar{B}_{b}, b\right]$, so $a=b$ since $\left[\bar{B}_{x}, x\right]$ is extensional. Thus $v=w$ by the same argument as in the previous case.

Hence the extensional quotient of $[\bar{B}, v\lceil h\rceil]$ is a multigraph.

Definition 4.70 If $B$ is a subimage of $[H, h]$, define $\left[Q^{B}, q^{B}\right] \in \mathcal{M}$ to be the extensional quotient of $[\bar{B}, v\lceil h\rceil]$.

Definition 4.71 If $B$ is a subimage of $[H, h]$, denote the quotient map by $\pi: \operatorname{Dom}[\bar{B}, v\lceil h\rceil] \rightarrow \operatorname{Dom}\left[Q^{B}, q^{B}\right]$.

Then the primary projection of $B$ is

$$
\begin{aligned}
& \left\{\left\langle\left\lfloor q_{1} \cdots q_{2 n+1}\right\rfloor,\left\lfloor h_{1} \cdots h_{2 n+1}\right\rfloor\right\rangle\right. \\
& \left.\quad \in \exp \left[Q^{B}, q^{B}\right] \times B:(\forall 1 \leq i \leq 2 n+1) q_{i}=\pi v\left\lceil h_{1} \cdots h_{i}\right\rceil\right\} .
\end{aligned}
$$


Lemma 4.72 For any $\left\lfloor q_{1} \cdots q_{2 n+1}\right\rfloor \in \exp \left[Q^{B}, q^{B}\right]$ there exists $\left\lfloor h_{1} \cdots h_{2 n+1}\right\rfloor$ such that $\left\langle\left\lfloor q_{1} \cdots q_{2 n+1}\right\rfloor,\left\lfloor h_{1} \cdots h_{2 n+1}\right\rfloor\right\rangle$ is in the primary projection.

Proof Suppose $q_{2 i-1}=\pi v\left\lceil h_{1} \cdots h_{2 i-1}\right\rceil$ for some $\left\lfloor h_{1} \cdots h_{2 i-1}\right\rfloor \in B$, or suppose $i=1$ and $q_{2 i-1}=\pi \nu\lceil h\rceil$.

Now $\left\langle q_{2 i-1}, q_{2 i}, q_{2 i+1}\right\rangle \in Q^{B}$, and by Definition 4.66 the only edges of $\bar{B}$ to come out of $v\left\lceil h_{1} \cdots h_{2 i-1}\right\rceil$ are

$$
\left\{\left\langle v\left\lceil h_{1} \cdots h_{2 i-1}\right\rceil, v\left\lceil h_{1} \cdots h_{2 i}\right\rceil, v\left\lceil h_{1} \cdots h_{2 i+1}\right\rceil\right\rangle:\left\lfloor h_{1} \cdots h_{2 i+1}\right\rfloor \in B\right\} .
$$

So there are $h_{2 i}, h_{2 i+1}$ such that $q_{2 i}=\pi v\left\lceil h_{1} \cdots h_{2 i}\right\rceil, q_{2 i+1}=\pi v\left\lceil h_{1} \cdots h_{2 i+1}\right\rceil$, and $\left\lfloor h_{1} \cdots h_{2 i+1}\right\rfloor \in B$.

Since $q_{1}=\pi v\lceil h\rceil$, we can build the required edge chain $\left\lfloor h_{1} \cdots h_{2 n+1}\right\rfloor$ by induction on $i$.

Lemma 4.73 The primary projection $P$ of $B$ is a projection to $\exp [H, h]$, and its range is precisely $B$.

Proof Let $\left\langle\left\lfloor q_{1} \cdots q_{2 n+1}\right\rfloor,\left\lfloor h_{1} \cdots h_{2 n+1}\right\rfloor\right\rangle,\left\langle\left\lfloor q_{1} \cdots q_{2 n+1}\right\rfloor,\left\lfloor b_{1} \cdots b_{2 n+1}\right\rfloor\right\rangle \in P$.

For any $1 \leq i \leq n$, by definition of the quotient map and Lemma 4.68,

$$
\left[Q_{q_{2 i}}^{B}, q_{2 i}\right] \cong\left[\bar{B}_{\nu\left\lceil h_{1} \cdots h_{2 i}\right\rceil}, v\left\lceil h_{1} \cdots h_{2 i}\right\rceil\right] \cong\left[H_{h_{2 i}}, h_{2 i}\right] .
$$

Similarly $\left[Q_{q_{2 i}}^{B}, q_{2 i}\right] \cong\left[H_{b_{2 i}}, b_{2 i}\right]$, so $h_{2 i}=b 2 i$ since $[H, h]$ is extensional. By Lemma 4.56, $\left\lfloor h_{1} \cdots h_{2 n+1}\right\rfloor=\left\lfloor b_{1} \cdots b_{2 n+1}\right\rfloor$, so $P$ is the graph of a partial function. By Lemma 4.72, this function is defined on all of $\exp \left[Q^{B}, q^{B}\right]$, so we will denote it by $\varphi: \exp \left[Q^{B}, q^{B}\right] \rightarrow B$.

If $\varphi\left\lfloor q_{1} \cdots q_{2 n+1}\right\rfloor=\varphi\left\lfloor g_{1} \cdots g_{2 n+1}\right\rfloor$, then $\left[Q_{q_{2 i}}^{B}, q_{2 i}\right] \cong\left[H_{h_{2 i}}, h_{2 i}\right] \cong$ $\left[Q_{g_{2 i}}^{B}, g_{2 i}\right]$ for all $1 \leq i \leq n$, so $\left\lfloor q_{1} \cdots q_{2 n+1}\right\rfloor=\left\lfloor g_{1} \cdots g_{2 n+1}\right\rfloor$ by Lemma 4.56 again.

Thus $\varphi$ is injective, and we have already verified the second condition of Definition 4.58 above.

Suppose $\varphi\left\lfloor q_{1} \cdots q_{2 n-1}\right\rfloor=\left\lfloor h_{1} \cdots h_{2 n-1}\right\rfloor$ and $\varphi\left\lfloor q_{1} \cdots q_{2 n+1}\right\rfloor=\left\lfloor b_{1} \cdots b_{2 n+1}\right\rfloor$. Then $\left\lfloor b_{1} \cdots b_{2 n-1}\right\rfloor \in B$ and $\left[H_{h_{2 i}}, h_{2 i}\right] \cong\left[Q_{q_{2 i}}^{B}, q_{2 i}\right] \cong\left[H_{b_{2 i}}, b_{2 i}\right]$ for all

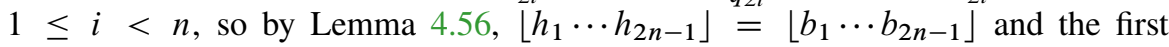
condition of Definition 4.58 holds.

By definition, $\varphi$ preserves chain length, so it is a projection.

Finally, for any $\left\lfloor h_{1} \cdots h_{2 n+1}\right\rfloor \in B$, let $q_{i}=\pi v\left\lceil h_{1} \cdots h_{i}\right\rceil$ for $1 \leq i \leq 2 n+1$. By Definition 4.66 we have the following edge sequence in $\bar{B}$ :

$$
\left\langle v\left\lceil h_{1} \cdots h_{2 i-1}\right\rceil, v\left\lceil h_{1} \cdots h_{2 i}\right\rceil, v\left\lceil h_{1} \cdots h_{2 i+1}\right\rceil\right\rangle: 1 \leq i \leq n .
$$

Hence $\left\lfloor q_{1} \cdots q_{2 n+1}\right\rfloor \in \exp \left[Q^{B}, q^{B}\right]$ so $\varphi\left\lfloor q_{1} \cdots q_{2 n+1}\right\rfloor=\left\lfloor h_{1} \cdots h_{2 n+1}\right\rfloor$ by definition. Therefore $\operatorname{ran} \varphi=B$.

Corollary 4.74 If $B$ is a subimage of $[H, h]$, then $\left[Q^{B}, q^{B}\right] \bar{C}[H, h]$.

Proof The corollary is proved by Lemmas 4.73 and 4.62 .

For completeness we include the next result, though we will not use it.

Lemma 4.75 If $A, B$ are subimages of $[H, h]$ such that $\left[Q^{A}, q^{A}\right] \cong\left[Q^{B}, q^{B}\right]$, then $A=B$. 
Proof Let $\psi: \operatorname{Dom}\left[Q^{A}, q^{A}\right] \rightarrow \operatorname{Dom}\left[Q^{B}, q^{B}\right]$ be an isomorphism.

Let $\varphi_{A}: \exp \left[Q^{A}, q^{A}\right] \rightarrow A$ and $\varphi_{B}: \exp \left[Q^{B}, q^{B}\right] \rightarrow B$ be the primary projections.

Suppose $\left\lfloor a_{1} \cdots a_{2 n+1}\right\rfloor \in A$; then $\left\lfloor a_{1} \cdots a_{2 n+1}\right\rfloor=\varphi_{A}\left\lfloor q_{1} \cdots q_{2 n+1}\right\rfloor$ for some $\left\lfloor q_{1} \cdots q_{2 n+1}\right\rfloor \in \exp \left[Q^{A}, q^{A}\right]$. But $\left\lfloor\psi\left(q_{1}\right) \cdots \psi\left(q_{2 n+1}\right)\right\rfloor \in \exp \left[Q^{B}, q^{B}\right]$, so let $\left\lfloor b_{1} \cdots b_{2 n+1}\right\rfloor=\varphi_{B}\left\lfloor\psi\left(q_{1}\right) \cdots \psi\left(q_{2 n+1}\right)\right\rfloor$. Then for any $1 \leq i \leq n$,

$$
\left[H_{a_{2 i}}, a_{2 i}\right] \cong\left[Q_{q_{2 i}}^{A}, q_{2 i}\right] \cong\left[Q_{\psi\left(q_{2 i}\right)}^{B}, \psi\left(q_{2 i}\right)\right] \cong\left[H_{b_{2 i}}, b_{2 i}\right] .
$$

By Lemma 4.56 we have $\left\lfloor a_{1} \cdots a_{2 n+1}\right\rfloor=\left\lfloor b_{1} \cdots b_{2 n+1}\right\rfloor \in B$. By the same argument any edge chain in $B$ is also in $A$, so $A=B$.

Lemma 4.76 If $[Q, q] \bar{\subset}[H, h]$, there exists a subimage $B$ of $[H, h]$ such that $[Q, q] \cong\left[Q^{B}, q^{B}\right]$.

Proof By Lemma 4.62 there is a projection $\psi: \exp [Q, q] \rightarrow \exp [H, h]$, so let $B=\operatorname{ran} \psi$. By Lemma 4.60, $B$ is closed under initial chains, so it is a subimage of $[H, h]$.

Let $\varphi: \exp \left[Q^{B}, q^{B}\right] \rightarrow B$ be the primary projection of $B$. By Lemma 4.61 both inverses $\psi^{-1}: B \rightarrow \exp [Q, q]$ and $\varphi^{-1}: B \rightarrow \exp \left[Q^{B}, q^{B}\right]$ are projections. Thus by Lemma 4.59 the composites $\psi^{-1} \varphi: \exp [Q, q] \rightarrow \exp \left[Q^{B}, q^{B}\right]$ and $\varphi^{-1} \psi: \exp \left[Q^{B}, q^{B}\right] \rightarrow \exp [Q, q]$ are also projections.

By Lemma 4.62 again $[Q, q] \bar{\subset}\left[Q^{B}, q^{B}\right]$ and $\left[Q^{B}, q^{B}\right] \bar{\subset}[Q, q]$, so $[Q, q] \cong$ $\left[Q^{B}, q^{B}\right]$ by Lemma 4.31 .

This means the set $\left\{\left[Q^{B}, q^{B}\right]: B\right.$ is a subimage of $\left.[H, h]\right\}$ contains one representative from each isomorphism class of multigraph $[Q, q] \bar{C}[H, h]$. Define a constant function $\delta$ on this set by $\delta\left[Q^{B}, q^{B}\right]=[\emptyset, 0]$. Note that $[\emptyset, 0]$ corresponds to the empty multiset in our interpretation of the language, which will give us the canonical power set of $[H, h]$ by supertransitivity.

Lemma 4.77 (Axiom of power set) $\quad$ If $[H, h] \in \mathcal{M}$, there exists $[D, d] \in \mathcal{M}$ such that

$$
(\forall[Q, q] \in \mathcal{M})\left([Q, q] \bar{\subset}[H, h] \Leftrightarrow\left([Q, q] \bar{\epsilon}[D, d] \wedge \frac{[H, h]}{[Q, q]} \cong[\emptyset, 0]\right)\right) .
$$

Proof We have proved that for any $[Q, q] \in \mathcal{M}$,

$$
[Q, q] \bar{\subset}[H, h] \Leftrightarrow\left(\exists\left[Q^{B}, q^{B}\right] \in \operatorname{dom} \delta\right)[Q, q] \cong\left[Q^{B}, q^{B}\right] .
$$

Therefore by Lemma 4.54 the result holds.

\subsection{The remaining axioms}

Lemma 4.78 (Axiom schema of multiplicity replacement) Let $[H, h] \in \mathcal{M}$ and $\varphi(x)$ be a formula in $\mathscr{L}_{\mathcal{M}}$ (see Definition 3.1) with parameters in $\mathcal{M}$. Suppose that for any $[Q, q] \bar{\epsilon}[H, h]$ there is a unique $[A, a] \in \mathcal{M}$ (up to isomorphism) such that $\mathcal{M} \models \varphi([Q, q],[A, a])$. Then there exists $[D, d] \in \mathcal{M}$ such that the following both hold:

$$
\begin{gathered}
(\forall[Q, q] \in \mathcal{M})([Q, q] \bar{\epsilon}[D, d] \Leftrightarrow[Q, q] \bar{\epsilon}[H, h]), \\
(\forall[Q, q] \bar{\epsilon}[D, d]) \varphi\left([Q, q], \frac{[D, d]}{[Q, q]}\right) .
\end{gathered}
$$


Proof By collection in $V$, there is a set $N$ such that

$$
(\forall[Q, q] \bar{\epsilon}[H, h])(\exists[A, a] \in N) \mathcal{M} \models \varphi([Q, q],[A, a]) .
$$

By comprehension in $V$ from $N$, there is a set $M \subset \mathcal{M}$ such that for all $[E, e] \in \mathcal{M}$,

$$
((\exists[A, a] \in M)[A, a] \cong[E, e]) \Leftrightarrow \mathcal{M} \models(\exists[Q, q] \bar{\epsilon}[H, h]) \varphi([Q, q],[E, e]) .
$$

Without loss of generality assume that all members of $M$ have disjoint domains and are disjoint from $\operatorname{Dom}[H, h]$. We construct a pointed hypergraph $[B, b]$ as follows.

- Let $B:=\bigcup\left\{H_{x}: x \in H^{-1} h\right\} \cup \bigcup\{A:[A, a] \in M\}$.

- Add to $B$ a new vertex $b$ and edges $\langle b, x, a\rangle$ for each pair $x \in H^{-1} h$ and $[A, a] \in M$ such that $\varphi\left(\left[H_{x}, x\right],[A, a]\right)$.

Then $[B, b]$ satisfies the condition in Lemma 4.22, so its extensional quotient $[D, d]$ is a multigraph. By the quotient lemma, it is straightforward to verify that $[D, d]$ is the required multigraph.

Lemma 4.79 (Axiom schema of comprehension) Let $[H, h] \in \mathcal{M}$ and $\varphi(x)$ be a formula in $\mathscr{L}_{\mathcal{M}}$ with parameters in $\mathcal{M}$. Then there exists $[D, d] \in \mathcal{M}$ such that the following both hold:

$$
\begin{gathered}
(\forall[Q, q] \in \mathcal{M})([Q, q] \bar{\epsilon}[D, d] \Leftrightarrow([Q, q] \bar{\epsilon}[H, h] \wedge \mathcal{M} \models \varphi([Q, q]))), \\
(\forall[Q, q] \bar{\epsilon}[D, d])\left(\frac{[D, d]}{[Q, q]} \cong \frac{[H, h]}{[Q, q]}\right) .
\end{gathered}
$$

Proof Let $A:=\left\{\left[H_{a}, a\right]: a \in H^{-1} h \wedge \mathcal{M} \models \varphi\left(\left[H_{a}, a\right]\right)\right\}$, and define $\delta: A \rightarrow \mathcal{M}$ by $\delta\left(\left[H_{a}, a\right]\right):=\frac{[H, h]}{\left[H_{a}, a\right]}$. Then $\delta$ sends isomorphic multigraphs to the same image, so by Lemma 4.54 we have $[D, d] \in \mathcal{M}$, as required.

With the presence of multiplicity replacement, the axiom of collection below implies the axiom of replacement as formulated in the theory MS.

Lemma 4.80 (Axiom of collection) $\quad$ Let $[H, h] \in \mathcal{M}$ and $\varphi(x, y)$ be a formula in $\mathscr{L}_{\mathcal{M}}$ with parameters in $\mathcal{M}$ such that

$$
(\forall[Q, q] \bar{\epsilon}[H, h])(\exists[A, a] \in \mathcal{M}) \mathcal{M} \models \varphi([Q, q],[A, a]) .
$$

Then there exists $[D, d] \in \mathcal{M}$ such that

$$
(\forall[Q, q] \bar{\epsilon}[H, h])(\exists[A, a] \bar{\epsilon}[D, d]) \mathcal{M} \models \varphi([Q, q],[A, a]) .
$$

Proof By collection in $V$, let $B$ be a set such that

$$
\left(\forall q \in H^{-1} h\right)(\exists[A, a] \in B) \mathcal{M} \models \varphi\left(\left[H_{q}, q\right],[A, a]\right) .
$$

By comprehension we have a set

$$
D:=\{[A, a] \in B \cap \mathcal{M}:(\exists[Q, q] \bar{\epsilon}[H, h]) \mathcal{M} \models \varphi([Q, q],[A, a])\} .
$$

Define $\delta$ on $D$ by $\delta([A, a]):=[H, h]$, and apply Lemma 4.54 .

Lemma 4.81 (Axiom of infinity) There is a well-ordered multigraph $\bar{\omega}$ that satisfies Axiom 3.3.1. 
Proof Let $\alpha_{0}:=[\emptyset, 0]$, an empty multiset in the sense of $\mathcal{M}$. If $\alpha_{n}=[A, a]$, define $\alpha_{n+1}:=[B, b]$ where $b \notin \operatorname{Dom}[A, a]$ and $B=A \cup\left\{\langle b, x, 0\rangle: x \in A^{-1} a\right\} \cup$ $\{b, a, 0\}$.

By induction on $n$, it is straightforward to show that $\alpha_{n} \bar{\epsilon} \mathcal{M}$ and

$$
\mathcal{M} \models \alpha_{n+1}=\alpha_{n} \cup\left\{\alpha_{n} \otimes \bar{\emptyset}\right\} .
$$

Define $\varphi$ on $\left\{\alpha_{n}: n \in \omega\right\}$ by $\varphi\left(\alpha_{n}\right)=[\emptyset, 0]$, and apply Lemma 4.54.

We have showed that $\mathcal{M}$ interprets MSR, whence we have the following.

Theorem 4.82 If ZF is consistent, then MSR is consistent.

Our model also satisfies the following antifoundation axiom (adapted from Aczel's antifoundation axiom in [1, p. 6]).

\section{Axiom 4.8.1 Multiset AFA}

If $H$ is a hypergraph (defined in the language of multiset theory using multiset relations and ordered pairs in the same manner as hypergraphs in set theory) such that

$$
\begin{aligned}
& (\forall a, b, c, d, e \bar{\epsilon} \operatorname{Dom} H)\left(\left(H(a, b, c) \wedge H(a, d, e) \wedge\left[H_{b}, b\right] \equiv\left[H_{d}, d\right]\right)\right. \\
& \left.\quad \Rightarrow\left[H_{c}, c\right] \equiv\left[H_{e}, e\right]\right)
\end{aligned}
$$

then there exists a unique function $\varphi$ such that $\operatorname{dom} \varphi=\operatorname{Dom} H$ and

$$
(\forall x \bar{\epsilon} \operatorname{Dom} H)(\forall a, b)\left(a \epsilon_{b} \varphi(x) \Leftrightarrow(\exists\langle x, y, z\rangle \bar{\epsilon} H)(\varphi(y)=a \wedge \varphi(z)=b)\right) .
$$

Lemma 4.83 Under the given interpretation, $\mathcal{M}$ is a model of multiset AFA.

Proof Suppose that $\mathcal{M}$ believes $[H, h]$ is a hypergraph satisfying the condition of multiset AFA. Define a hypergraph $G$ as

$$
\begin{aligned}
(\forall x, y, z & \in \operatorname{Dom}[H, h])(\langle x, y, z\rangle \in G \\
\quad \Leftrightarrow \mathcal{M} & \left.\models\left\langle\left[H_{x}, x\right],\left[H_{y}, y\right],\left[H_{z}, z\right]\right\rangle \bar{\epsilon}[H, h]\right) .
\end{aligned}
$$

Then for any $x \in \operatorname{Dom} G,\left[G_{x}, x\right]$ satisfies the conditions of Lemma 4.22, so its extensional quotient is a multigraph. Moreover, for all $x, y, z \in \operatorname{Dom} G$,

$$
\left(\mathcal{M} \models\left[G_{y}, y\right] \epsilon_{\left[G_{z}, z\right]}\left[G_{x}, x\right]\right) \Leftrightarrow\left(\mathcal{M} \models\left\langle\left[H_{x}, x\right],\left[H_{y}, y\right],\left[H_{z}, z\right]\right\rangle \bar{\epsilon}[H, h]\right) .
$$

For each $x \in \operatorname{Dom} G$ we can define a canonical $M_{x} \in \mathcal{M}$ such that

$$
\mathcal{M} \models M_{x}=\left\langle\left[H_{x}, x\right],\left[G_{x}, x\right]\right\rangle .
$$

Define a constant function $\psi:\left\{M_{x}: x \in \operatorname{Dom} G\right\} \rightarrow \mathcal{M}$ by $\varphi\left(M_{x}\right)=[\emptyset, 0]$. Then the supertransitivity lemma (Lemma 4.54) gives the required object in $\mathcal{M}$.

\section{A Model of Multiset Theory Where the Inclusion Relation is not Antisymmetric}

In any well-founded model of MS an induction on the recursive definition of $\bar{c}$ will show it to be antisymmetric; hence the axiom of foundation implies the axiom of subset in that case. However, as an application of the antifoundation property of our model $\mathcal{M}$, we will modify it slightly to obtain a model of MSR where $\bar{C}$ is not antisymmetric. 
5.1 Modified multigraphs Let $\mathcal{M}$ be the class of multigraphs as defined in Definition 4.20. In this section we will redefine multigraphs by strengthening the notions of a bisimulation as follows.

Definition 5.1 Let $\mathbb{A}:=[\langle 1,0,1\rangle, 1]$, that is, the pointed hypergraph with vertices 0 and 1 , where 1 is the point and 0 belongs to 1 with multiplicity 1 .

Definition 5.2 A relation $\sim \subset \operatorname{Dom}[G, g] \times \operatorname{Dom}[H, h]$ is a bisimulation between $[G, g]$ and $[H, h]$ if all of the following hold.

- It is a bisimulation in the old definition, that is, Definition 4.10.

- If $1 \sim x$ and $\left[G_{1}, 1\right]=\mathbb{A}$, then $1 \in \operatorname{Dom}\left[H_{x}, x\right]$ and $\left[H_{1}, 1\right]=\mathbb{A}$.

- If $x \sim 1$ and $\left[H_{1}, 1\right]=\mathbb{A}$, then $1 \in \operatorname{Dom}\left[G_{x}, x\right]$ and $\left[G_{1}, 1\right]=\mathbb{A}$.

Informally speaking, under this new definition $\mathbb{A}$ is no longer equivalent to its other isomorphic images. Nevertheless we can easily adapt the proofs of earlier results about bisimilarity to this new definition. From this point on we construct the model in exactly the same way as before except for a minor subtlety. First, we redefine the class $\mathcal{M}$ of multigraphs using the new definition of bisimulation, and we define relations on $\mathcal{M}$ to stand in for the identity and membership relations.

Definition 5.3 Say that $[H, h]$ is extensional if any nonempty bisimulation on $[H, h]$ is the identity.

Definition 5.4 Say that $[G, g] \equiv[H, h]$, that is, they are bisimilar, if there is a bisimulation $\sim$ between them such that $g \sim h$.

Definition 5.5 Say that $[G, g] \bar{\epsilon}[H, h]$ if there exists $x \in \operatorname{Dom}[H, h]$ such that $[G, g] \equiv\left[H_{x}, x\right]$.

If $[G, g] \equiv[H, h]$, then it is easy to see that their extensional quotients are isomorphic. However, unlike in the old model, even isomorphic multigraphs need not be bisimilar. For example, let $[G, g]$ be a distinct but isomorphic copy of $\mathbb{A}$; then the extensional quotients of $[G, g]$ and $\mathbb{A}$ are just themselves, but there is no bisimulation between them that relates the top vertices to each other (since by Definition 5.2 we would then have $g=1$ and $[G, g]=\mathbb{A}$ ). Nevertheless we can easily check that $\equiv$ is an equivalence relation that respects $\bar{\epsilon}$; thus when proving previous results in the new model we need to replace all instances of isomorphism with the bisimilarity relation.

\subsection{The axioms of MSR}

Remark 5.6 The proof of extensionality requires only trivial modification to check the case when $\mathbb{A}$ is involved.

Let us now address a small inconvenience. In the old model, given any collection of multigraphs we can easily create a copy in which all the multigraphs have disjoint domains. This was crucial in the proof of many axioms such as collection, where a large multigraph needs to be created. However, the special status of $\mathbb{A}$ in our new model means that we are longer allowed to replace it with isomorphic copies. Thus we have to slightly weaken the conditions of disjoint domains.

Definition 5.7 If $A \subset \mathcal{M}$ is a collection of multigraphs, say that $A$ is almost disjoint if the following hold for any $[G, g],[H, h] \in A$ : 
- if $1 \in \operatorname{Dom}[G, g], 1 \in \operatorname{Dom}[H, h]$, and $\left[G_{1}, 1\right]=\left[H_{1}, 1\right]=\mathbb{A}$, then $\operatorname{Dom}[G, g] \cap \operatorname{Dom}[H, h]=\{0,1\}$;

- otherwise $\operatorname{Dom}[G, g] \cap \operatorname{Dom}[H, h]=\emptyset$.

Say that $B$ is an almost disjoint copy of $A$ if $B$ is almost disjoint and there is a bijection $\pi: A \leftrightarrow B$ such that $[G, g] \equiv \pi[G, g]$ for all $[G, g] \in A$.

Note that this definition also works for classes if we allow the bijection to be a function class, but for our current purposes it suffices to consider only sets.

Lemma 5.8 For any collection $A \subset \mathcal{M}$ there is an almost disjoint copy.

Proof If $1 \notin \operatorname{Dom}[G, g]$ or $\left[G_{1}, 1\right] \neq \mathbb{A}$, define $\pi[G, g]$ as follows.

For any $a \in \operatorname{Dom}[G, g]$ let $\hat{a}:=\langle a,[G, g]\rangle$, then trivially $a \neq 0$ and $a \neq 1$.

Let $\hat{G}:=\{\langle\hat{a}, \hat{b}, \hat{c}\rangle:\langle a, b, c\rangle \in G\}$ and $\pi[G, g]:=[\hat{G}, \hat{g}]$. Then $\pi[G, g] \cong$ $[G, g]$ and the isomorphism is a bisimulation between them.

If $1 \in \operatorname{Dom}[G, g]$ and $\left[G_{1}, 1\right]=\mathbb{A}$, define $\hat{a}$ for $a \in \operatorname{Dom}[G, g]$ as above except that $\hat{0}=0$ and $\hat{1}=1$, and define $\pi[G, g]$ as above.

Then $\pi[G, g] \cong[G, g]$ is a bisimulation since both 0 and 1 are fixed.

It is straightforward to check that the range of $\pi$ is almost disjoint.

Remark 5.9 Let $A$ be an almost disjoint set of multigraphs, and let $H$ be the hypergraph obtained by taking the union of all hypergraphs in $A$. Then for any $[G, g] \in A,[G, g]=\left[H_{g}, g\right]$. Hence we can repeat the proof of supertransitivity, using almost disjoint multigraphs where disjoint multigraphs were needed.

The proofs of comprehension, multiplicity replacement, collection, and infinity thus carry over trivially. Now we give the updated definition of $\overline{\mathrm{C}}$.

Definition 5.10 Say that $[G, g] \bar{\subset}[H, h]$ if there is a relation $\triangleleft \subset \operatorname{Dom}[G, g] \times$ $\operatorname{Dom}[H, h]$ such that $g \triangleleft h$ and for all $x \in \operatorname{Dom}[G, g], y \in \operatorname{Dom}[H, h]$,

$$
x \triangleleft y \Rightarrow\left(\forall a \in G^{-1} x\right)\left(\exists b \in H^{-1} y\right)\left(\left[G_{a}, a\right] \equiv\left[H_{b}, b\right] \wedge G(x, a) \triangleleft H(y, b)\right) .
$$

Lemma 5.11 If $[G, g] \equiv[H, h],[P, p] \equiv[Q, q]$ and $[G, g] \bar{\subset}[P, p]$, then $[H, h] \bar{\subset}[Q, q]$. Furthermore, $\bar{\subset}$ is reflexive, transitive, and

$$
[G, g] \bar{\subset}[H, h] \Leftrightarrow(\forall[Q, q] \bar{\epsilon}[G, g])\left([Q, q] \bar{\epsilon}[H, h] \wedge \frac{[G, g]}{[Q, q]} \bar{\subset} \frac{[H, h]}{[Q, q]}\right) .
$$

Proof For the first part let $\sim_{1}, \sim_{2}$ be the bisimulations involved, and let $\triangleleft$ witness $[G, g] \bar{\subset}[P, p]$. Then the following relation witnesses $[H, h] \bar{\subset}[Q, q]$ :

$$
x \prec y \Leftrightarrow d f(\exists a \in \operatorname{Dom}[G, g])(\exists b \in \operatorname{Dom}[P, p])\left(a \sim_{1} x \wedge b \sim_{2} y \wedge a \triangleleft b\right) .
$$

The rest of the proof is mostly the same as for Lemma 4.31 .

Note that in Lemma 4.31 we constructed a bisimulation explicitly to show that $\bar{C}$ is antisymmetric, but that construction fails to be a bisimulation in the new definition. In fact $\bar{C}$ is no longer antisymmetric.

Lemma 5.12 We have

$$
(\exists[G, g],[H, h] \in \mathcal{M})([G, g] \bar{\subset}[H, h] \wedge[H, h] \bar{\subset}[G, g] \wedge[G, g] \not \equiv[H, h]) .
$$


Proof Let $[G, g]=\mathbb{A}$, and let $[H, h]$ be a distinct but isomorphic copy. Since the empty multigraph $[\varnothing, 0]$ is equivalent to any isomorphic copy of itself, the isomorphism witnesses both $[G, g] \bar{\subset}[H, h]$ and $[H, h] \bar{\subset}[G, g]$, but clearly $[G, g] \not \equiv[H, h]$ (since by Definition 5.2 we would have $h=1$ and $[H, h]=\mathbb{A}$ ).

The axiom of union proceeds as before, and the proof of power set can be adapted using almost disjointed multisets and making special cases for the occurrence of $\mathbb{A}$. Finally the multigraph constructed in the proof of Lemma 4.81 still satisfies the updated definition of multigraph and proves infinity in this new model. Therefore we have proved the following.

Theorem 5.13 It is consistent with the rest of our multiset theory that the inclusion relation is not antisymmetric.

\section{Index of Notation and Terms}

$\bar{\epsilon} 300$
$\bar{\complement} 301$
$\frac{x}{y} 300$
$H^{-1}$ (hypergraph) 310
$H^{-2}$ (hypergraph) 310
$H^{-1}(x)$ (hypergraph) 310
$H^{-2}(x)$ (hypergraph) 310
$H_{x}$ (hypergraph) 311
$x_{H}$ (hypergraph) 311
$H(x, y)$ (hypergraph) 315
$H(x, y, z)$ (hypergraph) 310
$\mathscr{L}_{\mathcal{M}} 300$
$\operatorname{Rep}_{\varphi} x 302$

\author{
$T C(x) 307$ \\ axiom schema of multiplicity replace- \\ ment 326 \\ bisimulation 311 \\ closed multiset 304 \\ Core 307 \\ $\exp$ (multiset) 321 \\ function (multiset) 304 \\ MS 307 \\ MSR 309 \\ ordinal (multiset) 304 \\ relation (multiset) 304 \\ supertransitivity lemma 320
}

\section{References}

[1] Aczel, P., Non-Well-Founded Sets, vol. 14 of CSLI Lecture Notes, Stanford University Center for the Study of Language and Information, Stanford, 1988. Zbl 0668.04001. MR 0940014. 311, 328

[2] Blizard, W. D., "Multiset theory," Notre Dame Journal of Formal Logic, vol. 30 (1989), pp. 36-66. MR 0990203. DOI 10.1305/ndjfl/1093634995. 299, 302, 308

[3] Blizard, W. D., "Negative membership," Notre Dame Journal of Formal Logic, vol. 31 (1990), pp. 346-68. MR 1072070. DOI 10.1305/ndjfl/1093635499. 304

[4] Blizard, W. D., "The development of multiset theory," Modern Logic, vol. 1 (1991), pp. 319-52. MR 1112352. 299

[5] Blizard, W. D., "Dedekind multisets and function shells," Theoretical Computer Science, vol. 110 (1993), pp. 79-98. MR 1208660. DOI 10.1016/0304-3975(93)90351-S. 299, 308

[6] D'Agostino, G., and A. Visser, "On non-well-founded multisets: Scott collapse in the Multiworld" in JFAK: Essays Dedicated to Johan van Benthem on the Occasion of His 50th Birthday, edited by J. Gerbrandy, M. Marx, M. Rijke, and Y. Venema, CD-ROM, Vossiuspers AUP, Amsterdam, 1999. 300, 311, 314 
[7] D'Agostino, G., and A. Visser, "Finality regained: A coalgebraic study of Scott-sets and multisets," Archive for Mathematical Logic, vol. 41 (2002), pp. 267-98. MR 1901189. DOI 10.1007/s001530100110. 311, 314

[8] Hinnion, R., "Sur la théorie des ensembles de Quine," Ph.D. dissertation, Université Libre de Bruxelles, Brussels, 1975. 311

[9] Holmes, M. R., Elementary Set Theory with a Universal Set, vol. 10 of Cahiers du Centre de logique, Academia, Louvain-la-Neuve, Belgium, 1998. MR 1759289. 311

[10] Mathias, A. R. D., "Weak systems of Gandy, Jensen, and Devlin," pp. 149-224 in Set Theory, edited by J. Bagaria and S. Todorcevic, Trends in Mathematics, Birkhäuser, Basel, 2006. Zbl 1113.03046. MR 2267149. DOI 10.1007/3-7643-7692-9_6. 310

[11] Tarski, A., "A lattice-theoretical fixpoint theorem and its applications," Pacific Journal of Mathematics, vol. 5 (1955), pp. 285-309. MR 0074376. 313

41 Ruth Bagnall Court

Coleridge Road

Cambridge CB1 3NU

United Kingdom

meobet@gmail.com 